\title{
Article \\ Finite Element Study of Bio-Convective Stefan Blowing Ag-MgO/Water Hybrid Nanofluid Induced by Stretching Cylinder Utilizing Non-Fourier and Non-Fick's Laws
}

\author{
Puneet Rana ${ }^{1,+}$, Vinita Makkar ${ }^{2,+}$ and Gaurav Gupta ${ }^{1, *}$ \\ 1 School of Mathematical Sciences, College of Science and Technology, Wenzhou-Kean University, \\ Wenzhou 325060, China; prana@kean.edu or puneetranaiitr@gmail.com \\ 2 Department of Basic and Applied Sciences, School of Engineering and Sciences, GD Goenka University, \\ Gurgaon 122103, India; vini252011makkar@gmail.com \\ * Correspondence: ggupta@kean.edu \\ + These authors contributed equally to this work.
}

check for updates

Citation: Rana, P.; Makkar, V.; Gupta, G. Finite Element Study of Bio-Convective Stefan Blowing Ag-MgO/Water Hybrid Nanofluid Induced by Stretching Cylinder Utilizing Non-Fourier and Non-Fick's Laws. Nanomaterials 2021, 11, 1735. https: / / doi.org/10.3390/ nano11071735

Academic Editors: Simona Barison, Filippo Agresti, Laura Fedele,

David Cabaleiro and

Manuel M. Piñeiro

Received: 2 May 2021

Accepted: 25 June 2021

Published: 30 June 2021

Publisher's Note: MDPI stays neutral with regard to jurisdictional claims in published maps and institutional affiliations.

Copyright: (c) 2021 by the authors. Licensee MDPI, Basel, Switzerland. This article is an open access article distributed under the terms and conditions of the Creative Commons Attribution (CC BY) license (https:/ / creativecommons.org/licenses/by/ $4.0 /)$.

\begin{abstract}
In the present framework, an analysis on nanofluid magneto-transport phenomena over an extending cylinder influenced by gyrotactic behavior of algal suspension, is made using the CattaneoChristov heat flux (non-Fourier) and mass flux (non-Fick's) concept in modified Buongiorno's model. Two dimensional incompressible MHD hybrid nanofluid which comprises chemically reactive hybrid nanomaterials (Ag-MgO NPs) and Stefan blowing effect along with multiple slips is considered. The experimental correlations with their dependency on initial nanoparticle volume fraction are used for viscosity and thermal conductivity of nanofluids. Similarity transformation is used to convert the governing PDE's into non-linear ODE's along with boundary conditions, which are solved using the Galerkin Finite Element Method (GFEM). The mesh independent test with different boundary layer thickness $\left(\xi_{\infty}\right)$ has been conducted by taking both linear and quadratic shape functions to achieve a optimal desired value. The results are calculated for a realistic range of physical parameters. The validation of FEM results shows an excellent correlation with MATLAB $b v p 5 c$ subroutine. The warmth exhibitions are assessed through modified version of Buongiorno's model which effectively reflects the significant highlights of Stefan blowing, slip, curvature, free stream, thermophoresis, Brownian motion and bio-convection parameters. The present study in cylindrical domain is relevant to novel microbial fuel cell technologies utilizing hybrid nanoparticles and concept of Stefan blowing with bioconvection phenomena.
\end{abstract}

Keywords: hybrid nanofluid; cylinder; stefan blowing; FEM; non-fourier; bioconvection

\section{Introduction}

A rapid progress has urged researchers' attention over flow regime towards stretched cylinder. Fluid flow by a directly or non-straightly extending chamber assumes a critical part and have huge applications in assembling and creation measures including metal turning, creation of glass filaments, elastic sheet formation, wire drawing, expulsion of polymer sheets, petrol businesses, polymer preparing and so forth. The progression of quiet liquid over a moving surface was first broke down by Sakiadis [1]. Since most recent 20 years, the investigation of nanofluid has asked the scientist's consideration because of their warmth transportation rate. Nanofluid comes into existence when we add a little amount of nano-sized particles to the base liquids. The term nanofluid was first instituted by Choi and Eastman [2]. In 2006, Buongiorno [3] explained two-component nanofluid modelling based on seven slip mechanisms which further utilized for heat transport investigation in nanofluid uniform layer by Kuznetsov and Nield [4]. Dhanai et al. [5] studied the variable slip effects over an inclined cylinder in presence of mixed convection. Swapna et al. [6] studied radiative effect over permeable stretching cylinder by using FE 
technique. Rana et al. [7] studied the energy dissipating effect of Viscoelastic Nanofluid Flow by using hp-FEM technique. Recently, Vinita and Poply [8] inspected influence of free stream velocity over stretching cylinder in MHD nanofluids. Goyal et al. [9] examined the GFEM examination in MHD nanofluid flow towards extending sheet and the FEM approach was implemented to solve system. Vinita et al. [10] explained the effect of variable slip flows in addition to thermal radiation in MHD nanofluids induced by non linear stretched surface. Vinita et al. [11] examined radiation effect on MHD free stream velocity nanofluid flow induced by stretchable cylinder in presence of chemically reactive species by applying RKF technique using ODE45 solver in MATLAB. Khan et al. [12] studied the applications of bio-convection nanofluid flow in presence of activation energy. The influence of Fick's and Fourier laws on MHD dusty Casson nanofluid in presence of heat source parameter was investigated by Ramzan et al. [13].

In different related conditions of the heat move measure, probably the best guideline is exemplary Fourier heat law which is utilized in traditional material science [14]. One of its chief drawbacks of the constituting condition of heat is that particular aggravation and at the same time, the possibility of determinism are tested over the whole entire medium. Numerous specialists have clarified the conduct of pseudo-plastic liquids by recommending various models, especially Carreau, Williamson, power law, Upper Convective Maxwell (UCM), Cattaneo-Christov (Non-Fourier) Model, and so on. The Cattaneo's thermal unwinding is the modifed type of Fourier's thermal conduction law [15]. For Oldroyd's super convection model, Christov [16] extended Cattaneo's law [15] to achieve the invariance of structural elements by adding rest periods. Along these lines, another model called Cattaneo-Christov was introduced based on heat flux. Recently, Kumar et al. [17] examined the CNT's based flow along with thermal convective conditions and Cattaneo-Christov (non-Fourier) model. Abid et al. [18] studied the effect of two distinctive nanoparticles $\mathrm{Cu}$ and $\mathrm{CuO}$ in two diverse part of the way ionized magneto-fluid over a straightly extending surface. Recently, the flow of the boundary layer and the heat transfer model with Cattaneo-Christov have been extensively investigated in many publications (see [19-23]).

Stagnation point nanofluid flow with Stefan blowing in presence of chemical reaction and heat radiation has been studied by Rana et al. [24] by following Homotopy analysis. Additionally, Rana et al. [25] analyzed the entropy generation impact along with Stefan blowing and slip flow induced by horizontal surface using Lie analysis approach and found that higher Reynolds number rises entropy generation number. Gowda et al. [26] investigated the Stefan blowing impact with magnetic dipole over stretching surface in ferromagnetic nanofluids by following RKF45 technique and showed that heat transportation of fluid is enhanced with Stefan blowing convective conditions. Impact of Stefan blowing over rotating disc in Maxwell nanofluids has been investigated by Mabood et al. [27] and found that higher thermal relaxation parameter falls temperature field. Recently, Gowda et al. [28] presented the Stefan effect with Cattaneo-Christov model in Sutterby nanofluids over rotating disc by extending Buongiorno's model in their study. Additionally, Madhukesh et al. [29] used non-Fourier heat flux model over curved stretching surface to investigate the impact of AA7072-AA7075/water-based hybrid nanofluid along with Newtonian heating when temperature at the wall is constant. In 2021, two dimensional laminar flow of non-Newtonian Marangoni nanofluids in presence of activation energy along with chemical reactions has been presented by Gowda et al. [30] and they showed that heat transfer rate declines with larger Marangoni number. Very recently, Yusuf et al. [31] studied the bio-convective entropy generation effect induced by inclined plate in presence of Williamson nanofluid.

To achieve stability of hybrid nanofluids, the proper selection of NPs plays a significant role for base fluids. Various examinations have been directed to test the qualities of crossover nanofluids. Dominant parts of the analysts have announced empowering results. Hybrid nanofluids have been found to have streamlined qualities, demonstrating them to be appropriate for the heavenly bodies that require great warm, optical, and rheological attributes of working liquid. Flow caused by $\mathrm{Al}_{2} \mathrm{O}_{3}$ water-based nanofluid by shrinking 
cylinder in the presence of slip conditions was examined by Rana et al. [32] by following Buongiorno's model. Esfe et al. [33] explained that hybrid nanofluid have better influence on thermal conductivity than single particle nanofluids. Following segment sums up significant properties such as optical, warm, rheological, and morphological properties of mixture nanofluids according to revealed in the latest examination articles presented by Shah and Ali [34]. MHD hybrid nanofluid flow in annulus among concentric cylinders was studied by Rana [35]. A numerical investigation was done by Aminian et al. [36] to study the impact of Magnetic by extending cylinder in hybrid nanofluids. Gul et al. [37] showed the impact of magnetic dipole over stretching surface in hybrid nanofluid flow. Reddy et al. [38] explained the effect of CCHFM over hybrid dusty nanofluids. Khashi'ie et al. [39] examined hybrid nanofluid flow with prescribed surface heat flux Past a shrinking cylinder. Tassaddiq [40] examined the influence of CCHFM on MHD hybrid nanofluid flow in presence of viscous and Ohmic dissipation and found that macro velocity falls for higher values of Hartmann number and micropolar factor. In recent years, hybrid nanofluid flow of the boundary layer and the heat transfer model with Cattaneo-Christov in different circumstances have been extensively investigated in many publications [41-43].

An important point of current research is the outstanding investigation of hybrid nanofluid flow through CCHFM along with Stefan blowing, mass flux, chemical reaction and gyrotactic micro-organism over a stretched cylinder. The ruling PDEs are converted as ODE's to obtain numerical solution using Finite Element technique with different shape functions. The results are also compared with MATLAB finite difference $b v p 5 c$ function. A catalog who works well for the physical steps to solve this model are presented and correlation between flow and temperatures represented by tables and graphs. Finally, the physical quantities of interest are presented for various controlling parameters in the form of contours.

\section{Nano-Materials and Modeling}

Two dimensional incompressible MHD hybrid nanofluid (Ag-MgO/water) flow with gyrotactic micro-organisms in presence of chemically reactive species towards an extended surface with free stream velocity $\left(U_{\infty}\right)$ has been taken into account (See Figure 1$)$. The flow field is impacted by the gradient of nanoparticle volume fraction at the wall (Stefan blowing phenomena) with low magnetic Reynolds number. The cylinder is stretched with stretching velocity $u_{w}=a x / L$ in direction of $x$-axis whereas magnetic force has been employed in the direction of radial axis. Additionally, free stream motile organisms, nanoparticle volume fraction and temperature are expressed by $N_{\infty}, C_{\infty}$ and $T_{\infty}$ respectively. The governing equations for nanofluid are depicted as:

$$
\begin{gathered}
\frac{\partial}{\partial x}(r u)+\frac{\partial}{\partial r}(r v)=0 \\
u \frac{\partial u}{\partial x}+v \frac{\partial u}{\partial r}=\frac{\mu_{h n f}}{\rho_{h n f}}\left(\frac{\partial^{2} u}{\partial r^{2}}+\frac{1}{r} \frac{\partial u}{\partial r}\right)-\frac{\sigma_{h n f} B_{0}^{2}}{\rho_{h n f}}\left(u-U_{\infty}\right)+U_{\infty} \frac{\partial U_{\infty}}{\partial x} \\
u \frac{\partial T}{\partial x}+v \frac{\partial T}{\partial r}+\lambda_{E}\left[v \frac{\partial v}{\partial r} \frac{\partial T}{\partial r}+v \frac{\partial u}{\partial r} \frac{\partial T}{\partial x}+u \frac{\partial v}{\partial x} \frac{\partial T}{\partial r}+u \frac{\partial u}{\partial x} \frac{\partial T}{\partial x}+2 u v \frac{\partial^{2} T}{\partial x \partial r}+v^{2} \frac{\partial^{2} T}{\partial r^{2}}+u^{2} \frac{\partial^{2} T}{\partial x^{2}}\right] \\
=\frac{k_{h n f}}{(\rho c)_{h n f}}\left(\frac{\partial^{2} T}{\partial r^{2}}+\frac{1}{r} \frac{\partial T}{\partial r}\right)+\frac{(\rho c)_{p}}{(\rho c)_{h n f}}\left[D_{B} \frac{\partial C}{\partial r} \frac{\partial T}{\partial r}+\frac{D_{T}}{T_{\infty}}\left(\frac{\partial T}{\partial r}\right)^{2}\right]
\end{gathered}
$$




$$
\begin{array}{r}
u \frac{\partial C}{\partial x}+v \frac{\partial C}{\partial r}+\lambda_{C}\left[v \frac{\partial v}{\partial r} \frac{\partial C}{\partial r}+v \frac{\partial u}{\partial r} \frac{\partial C}{\partial x}+u \frac{\partial v}{\partial x} \frac{\partial C}{\partial r}+u \frac{\partial u}{\partial x} \frac{\partial C}{\partial x}+2 u v \frac{\partial^{2} C}{\partial x \partial r}+v^{2} \frac{\partial^{2} C}{\partial r^{2}}+u^{2} \frac{\partial^{2} C}{\partial x^{2}}\right] \\
=D_{B}\left(\frac{\partial^{2} C}{\partial r^{2}}+\frac{1}{r} \frac{\partial C}{\partial r}\right)+\frac{D_{T}}{T_{\infty}}\left(\frac{\partial^{2} T}{\partial r^{2}}+\frac{1}{r} \frac{\partial T}{\partial r}\right)-K_{r}\left(C-C_{\infty}\right) \\
u \frac{\partial N}{\partial x}+v \frac{\partial N}{\partial r}+\frac{d W_{C}}{C_{w}-C_{\infty}}\left[\frac{\partial}{\partial r}\left(N \frac{\partial C}{\partial r}\right)\right]=D_{N}\left(\frac{\partial^{2} N}{\partial r^{2}}+\frac{1}{r} \frac{\partial N}{\partial r}\right)-K_{r}\left(N-N_{\infty}\right)
\end{array}
$$

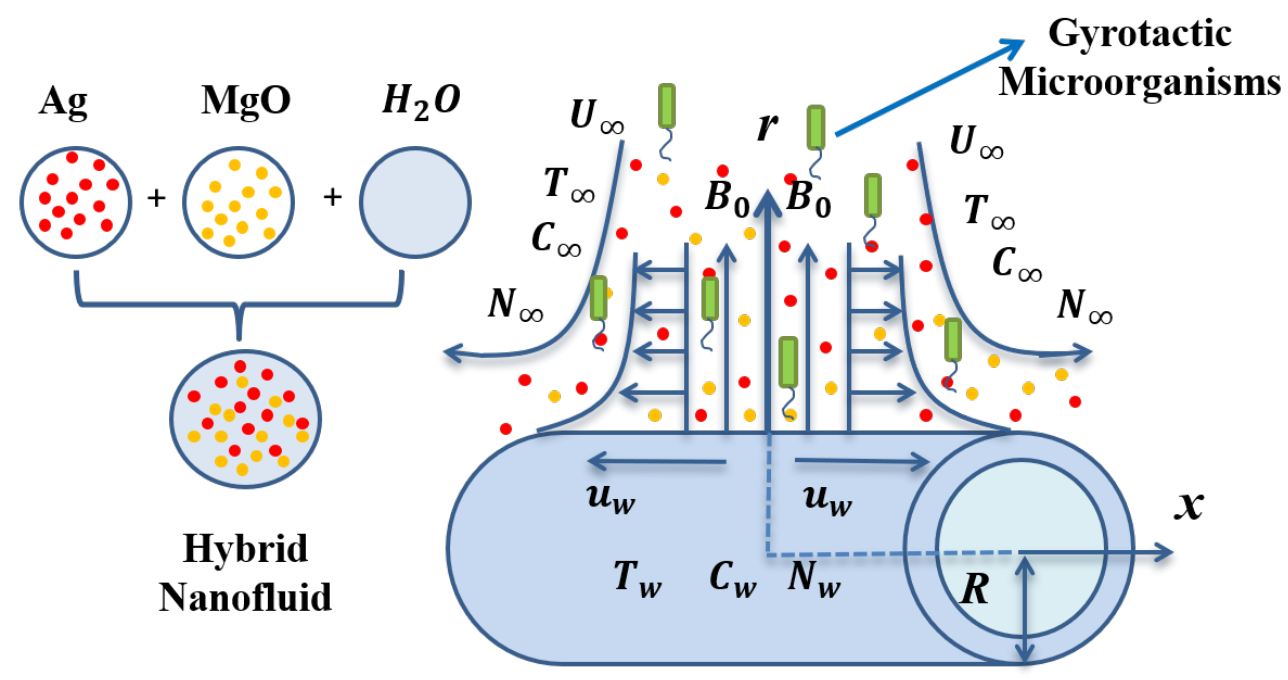

Figure 1. Physical model with co-ordinate system.

Here $v$ and $u$ represents radial velocity and horizontal velocity. Additionally, $T_{\infty}$ stands for ambient temperature, $D_{B}$ stands for Brownian diffusion coefficient, $\lambda_{E}$ denoted the thermal relaxation parameter, $T$ stands for temperature, $\lambda_{C}$ stands for nanoparticle volume fraction relaxation parameter, $D_{T}$ stands for thermophoresis diffusion coefficient, $K_{r}$ is the chemical reaction parameter, $C$ stands for nanoparticle volume fraction, $B_{0}$ stands for magnetic field intensity, $\sigma_{h n f}$ stands for electrical conductivity, $v_{h n f}$ stands for kinematic viscosity and $U_{\infty}$ stands for free stream velocity. In Equation (5), $d$ is constant, $W_{c}$ is maximum swimming speed of micro-organisms in hybrid-nanofluid and $D_{N}$ is diffusivity of micro-organisms.

Associated boundary conditions:

$$
\begin{array}{r}
u=u_{w}+N_{1} \frac{\partial u}{\partial r}, \quad v=-\frac{D_{B}}{1-C_{w}}\left(\frac{\partial C}{\partial r}\right), \quad T=T_{w}+N_{2}\left(\frac{\partial T}{\partial r}\right), \quad C=C_{w}+N_{3}\left(\frac{\partial C}{\partial r}\right), \\
N=N_{w}+N_{4}\left(\frac{\partial N}{\partial r}\right) \quad \text { at } \quad r=R, \\
\text { and } u \rightarrow U_{\infty}=\frac{b x}{L}, \quad T \rightarrow T_{\infty}, \quad C \rightarrow C_{\infty}, \quad N \rightarrow N_{\infty} \text { as } \quad r \rightarrow \infty
\end{array}
$$

The thermophysical properties of basefluid and nanoparticles $(A g$ and $M g O)$ are shown in Table 1. The correlations for electrical conductivity, heat capacitance, density, dynamic viscosity and thermal conductivity of $\mathrm{Ag}-\mathrm{MgO} /$ water hybrid nanofluid with the particle diameter of $40 \mathrm{~nm}(\mathrm{MgO})$ and $25 \mathrm{~nm}(\mathrm{Ag})$ and nanoparticle volume fraction (50\% $\mathrm{Ag}$ and $50 \% \mathrm{MgO}$ by volume), are specified as [44-46]: 


$$
\begin{gathered}
\sigma_{r}=\frac{\sigma_{h n f}}{\sigma_{f}}=\left[\frac{1+3\left(\frac{\sigma}{\sigma_{f}}-1\right)\left(\phi_{A g}+\phi_{M g O}\right)}{\left(\frac{\sigma}{\sigma_{f}}+2\right)-\left(\frac{\sigma}{\sigma_{f}}-1\right)\left(\phi_{A g}+\phi_{M g O}\right)}\right] \\
(\rho c)_{r}=\frac{(\rho c)_{h n f}}{(\rho c)_{f}}=\left(1-\phi_{A g}-\phi_{M g O}\right)+\phi_{A g} \frac{(\rho c)_{A g}}{(\rho c)_{f}}+\phi_{M g O} \frac{(\rho c)_{M g O}}{(\rho c)_{f}} \\
\rho_{r}=\frac{\rho_{h n f}}{\rho_{f}}=\left(1-\phi_{A g}-\phi_{M g O}\right)+\phi_{A g} \frac{\rho_{A g}}{\rho_{f}}+\phi_{M g O} \frac{\rho_{M g O}}{\rho_{f}} \\
\mu_{r}=\frac{\mu_{h n f}}{\mu_{f}}=\left[1+32.795 \phi_{1}-7214 \phi_{1}{ }^{2}+714600 \phi_{1}{ }^{3}-0.1941 \times 10^{8} \phi_{1}{ }^{4}\right] ; 0 \leq \phi_{1} \leq 0.02 \\
k_{r}=\frac{k_{h n f}}{k_{f}}=\left[\frac{0.1747 \times 10^{5}+\phi_{1}}{0.1747 \times 10^{5}-0.1498 \times 10^{6} \phi_{1}+0.1117 \times 10^{7} \phi_{1}{ }^{2}+0.1997 \times 10^{8} \phi_{1}^{3}}\right] ; \\
0 \leq \phi_{1} \leq 0.03
\end{gathered}
$$

Table 1. Thermophysical properties of basefluid and nanoparticles (Ag and MgO).

\begin{tabular}{cccc}
\hline & $\boldsymbol{A g}$ & $\boldsymbol{M g O}$ & Pure Water \\
\hline$\rho\left(\mathrm{Kg} / \mathrm{m}^{3}\right)$ & 10,500 & 3560 & 997.1 \\
$c(\mathrm{~J} / \mathrm{Kg} \mathrm{K})$ & 235 & 955 & 4179 \\
$k(\mathrm{~W} / \mathrm{m} \mathrm{K})$ & 429 & 45 & 0.62 \\
$\sigma(\mathrm{S} / \mathrm{m})$ & $6.21 \times 10^{7}$ & $\approx 10^{-8}$ & 0.05 \\
$\mu(\mathrm{Kg} / \mathrm{m} \mathrm{S})$ & - & - & $8.55 \times 10^{-4}$ \\
\hline
\end{tabular}

Additionally, the similarity variables are specified by following:

$\xi=\frac{r^{2}-R^{2}}{2 R}\left(\frac{u_{w} \rho_{f}}{x u_{f}}\right)^{\frac{1}{2}}, \quad \psi=\left(\frac{u_{w} x \mu_{f}}{\rho_{f}}\right)^{\frac{1}{2}} R f(\xi), \quad \theta(\xi)=\frac{T-T_{\infty}}{T_{w}-T_{\infty}} \quad \Phi(\xi)=\frac{C-C_{\infty}}{C_{w}-C_{\infty}}$

and $\chi(\xi)=\frac{N-N_{\infty}}{N_{w}-N_{\infty}}$

Inserting (12) into Equations (2)-(5), we have a system of the following differential equations:

$$
\begin{gathered}
\mu_{r}\left[(1+2 \xi \gamma) f^{\prime \prime \prime}+2 \gamma f^{\prime \prime}\right]+\rho_{r}\left[f f^{\prime \prime}-f^{\prime 2}+\epsilon^{2}\right]-\sigma_{r} M^{2}\left(f^{\prime}-\epsilon\right)=0 \\
k_{r}\left[(1+2 \xi \gamma) \theta^{\prime \prime}+2 \gamma \theta^{\prime}\right]+\operatorname{Pr} N b(1+2 \xi \gamma) \theta^{\prime} \Phi^{\prime}+\operatorname{Pr} N t(1+2 \xi \gamma) \theta^{\prime 2} \\
+(\rho c)_{r} \operatorname{Pr}\left[f \theta^{\prime}-\alpha_{t}\left(f^{2} \theta^{\prime \prime}+f f^{\prime} \theta^{\prime}\right)\right]=0 \\
(1+2 \xi \gamma) \Phi^{\prime \prime}+L e f \Phi^{\prime}+2 \gamma \Phi^{\prime}-L e \alpha_{c}\left(f^{2} \Phi^{\prime \prime}+f f^{\prime} \Phi^{\prime}\right) \\
+(1+2 \xi \gamma) \frac{N t}{N b} \theta^{\prime \prime}+2 \gamma \frac{N t}{N b} \theta^{\prime}-L e C_{r} \Phi=0 \\
(1+2 \xi \gamma) \chi^{\prime \prime}+L b f \chi^{\prime}+2 \gamma \chi^{\prime}- \\
\operatorname{Pe}\left[(1+2 \xi \gamma) \chi \Phi^{\prime \prime}+\gamma \chi \Phi^{\prime}+\Omega_{1} \gamma \Phi^{\prime}+\Omega_{1}(1+2 \xi \gamma) \Phi^{\prime \prime}+(1+2 \xi \gamma) \chi^{\prime} \Phi^{\prime}\right]-L b C_{r} \chi=0
\end{gathered}
$$

with

$$
\begin{aligned}
& f^{\prime}(0)=1+\delta_{1} f^{\prime \prime}(0), f(0)=\frac{S b}{L e P r} \Phi^{\prime}(0), \theta(0)=1+\delta_{2} \theta^{\prime}(0), \Phi(0)=1+\delta_{3} \Phi^{\prime}(0), \\
& \chi(0)=1+\delta_{4} \chi^{\prime}(0) \\
& \text { and } f^{\prime}(\xi) \rightarrow \epsilon, \theta(\xi) \rightarrow 0, \Phi(\xi) \rightarrow 0, \chi(\xi) \rightarrow 0 \quad \text { as } \quad \xi \rightarrow \infty
\end{aligned}
$$


where crucial fluid dimensionless parameters are specified as:

$$
\begin{aligned}
& \alpha_{t}=\frac{a \lambda_{E}}{L}, \quad \alpha_{c}=\frac{a \lambda_{C}}{L}, \quad C_{r}=\frac{K_{r} L}{a}, \quad L e=\frac{\mu_{f}}{\rho_{f} D_{B}}, \quad N b=\frac{(\rho c)_{p} D_{B}\left(C_{w}-C_{\infty}\right)}{(\rho c)_{f} v_{f}}, \quad \epsilon=\frac{b}{a}, \\
& \operatorname{Pr}=\frac{\mu_{f}}{\rho_{f} \alpha_{f}}, \quad \gamma=\frac{1}{R} \sqrt{\frac{\mu_{f} L}{\rho_{f} a}}, \quad N t=\frac{(\rho c)_{p} D_{T}\left(T_{w}-T_{\infty}\right)}{(\rho c)_{f} T_{\infty} v_{f}}, \quad M^{2}=\frac{\sigma_{f} B_{0}{ }^{2} L}{\rho_{f} a}, \quad S b=\frac{C_{w}-C_{\infty}}{1-C_{w}}, \\
& P e=\frac{d W_{c}}{D_{N}}, \quad \Omega_{1}=\frac{N_{\infty}}{N_{w}-N_{\infty}}, \quad L b=\frac{\mu_{f}}{\rho_{f} D_{N}}, \delta_{i}=N_{i} \sqrt{\frac{c \rho_{f}}{L \mu_{f}}}(i=1, \ldots, 4), \alpha_{f}=\frac{k_{f}}{(\rho c)_{f}} .
\end{aligned}
$$

The skin friction coefficient $C f_{r}$, local Nusselt number $N u_{r}$, local Sherwood number $S h_{r}$ and local motile micro-organism number $M_{r}$ are defined as

$$
C f=\frac{\tau_{w}}{\rho U_{\infty}^{2}}, N u=\frac{x q_{w}}{k\left(T_{f}-T_{\infty}\right)}, S h=\frac{x q_{m}}{D_{B}\left(C_{w}-C_{\infty}\right)}, M o=\frac{x q_{n}}{D_{N}\left(N_{w}-N_{\infty}\right)},
$$

where the wall shear stress $\tau_{w}$, the local heat flux $q_{w}$, the local mass flux $q_{m}$ and local micro-organism mass flux $q_{n}$ as follows

$$
\tau_{w}=\mu_{h n f}\left(\frac{\partial u}{\partial r}\right)_{r=R}, q_{w}=-k_{h n f}\left(\frac{\partial T}{\partial r}\right)_{r=R}, q_{m}=-D_{B}\left(\frac{\partial C}{\partial r}\right)_{r=R}, q_{n}=-D_{N}\left(\frac{\partial N}{\partial r}\right)_{r=R},
$$

where $\mu_{h n f}$ is the dynamic viscosity of hybrid nanofluid. Using the variables (12), the skin friction coefficient, local Nusselt number, local Sherwood number and local mobile micro-organism density number are given below:

$$
\begin{aligned}
& C f_{r}=C f R e_{x}^{1 / 2}=\mu_{r} f^{\prime \prime}(0), N u_{r}=N u R e_{x}^{-1 / 2}=-k_{r} \theta^{\prime}(0), S h_{r}=S h R e_{x}^{-1 / 2}=-\Phi^{\prime}(0), \\
& M_{r}=M o R e_{x}^{-1 / 2}=-\chi^{\prime}(0),
\end{aligned}
$$

where $\operatorname{Re}_{x}=u_{w} x / v_{f}$ is the local Reynold number.

\section{Numerical Method}

The Galerkin finite element approach (GFEM) is one of the well-known numerical strategies to discover approximate solutions of ODE in addition to PDE system that includes complex boundary situations and/or complicated geometry. The working of finite element method are shown in Figure 2. To solve this system of non-linear differential Equations (13)-(16) following (Swapna et al. [6], Rana et al. [7], Goyal et al. [9]), the weak formulation procedure has been adopted and $\Sigma_{e}$ represents the typical linear/quadratic element that is created by weighted residual formulation having element coordinates $\left(\xi_{e}, \xi_{e+1}\right)$, is given by

$$
\begin{gathered}
\int_{\Sigma_{e}} w_{1}\left\{f^{\prime}-h\right\} d \xi=0 \\
\int_{\Sigma_{e}} w_{2}\left\{\frac{d}{d \xi}\left[\mu_{r}(1+2 \xi \gamma) h^{\prime}\right]+\rho_{r}\left[f h-h^{2}+\epsilon^{2}\right]-\sigma_{r} M(h-\epsilon)\right\} d \xi=0 \\
\int_{\Sigma_{e}} w_{3}\left\{\begin{array}{l}
\frac{d}{d \xi}\left[k_{r}(1+2 \xi \gamma) \theta^{\prime}\right]+\operatorname{Pr} N b(1+2 \xi \gamma) \theta^{\prime} \Phi^{\prime}+\operatorname{Pr} N t(1+2 \xi \gamma) \theta^{\prime 2} \\
+(\rho c)_{r} \operatorname{Pr}\left[f \theta^{\prime}-\alpha_{t} f \frac{d}{d \xi}\left[f^{\prime} \theta^{\prime}\right]\right.
\end{array}\right\} d \xi=0 \\
\int_{\Sigma_{e}} w_{4}\left\{\frac{d}{d \xi}\left[(1+2 \xi \gamma) \Phi^{\prime}\right]+L e f \Phi^{\prime}+\frac{N t}{N b} \frac{d}{d \xi}\left[(1+2 \xi \gamma) \theta^{\prime}\right]-L e\left[\alpha_{c} f \frac{d}{d \xi}\left[f^{\prime} \Phi^{\prime}\right]+C r \Phi\right]\right\} d \xi=0 \\
\int_{\Sigma_{e}} w_{5}\left\{\begin{array}{l}
\frac{d}{d \xi}\left[(1+2 \xi \gamma) \chi^{\prime}\right]+L b f \chi^{\prime}-\operatorname{Pe}\left[\frac{d}{d \xi}\left[(1+2 \xi \gamma)\left(\chi+\Omega_{1}\right) \Phi^{\prime}\right]-\gamma\left(\chi+\Omega_{1}\right) \Phi^{\prime}\right] \\
-L b C r \chi
\end{array}\right\} d \xi=0
\end{gathered}
$$




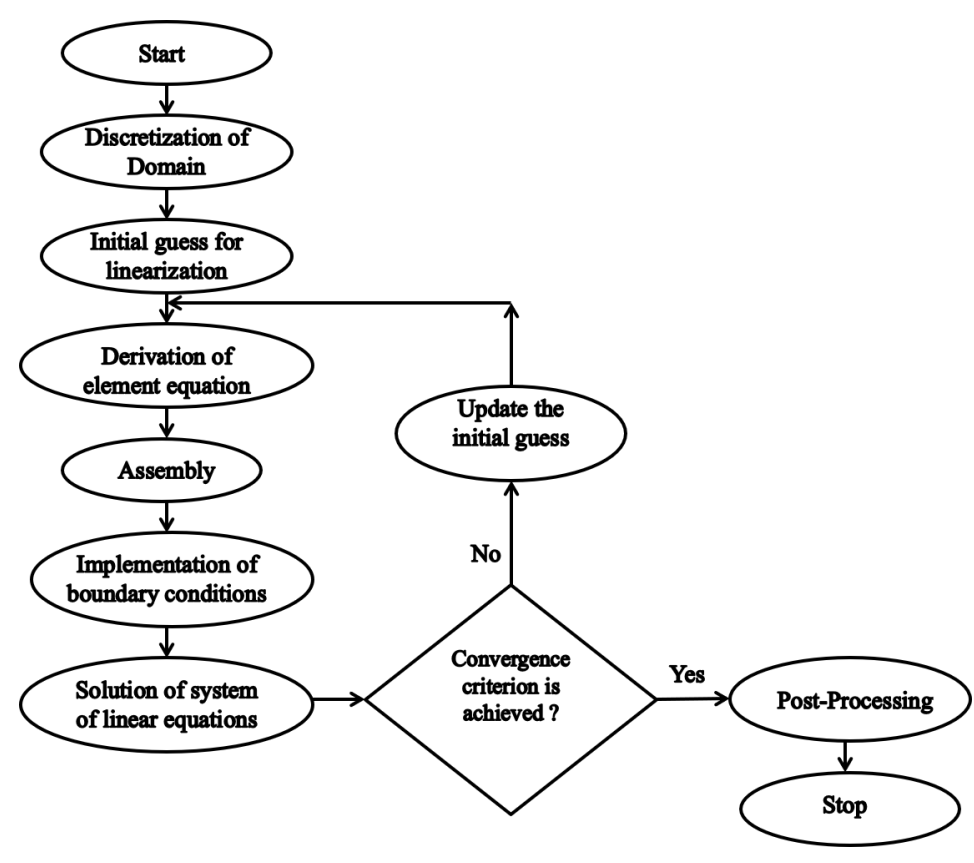

Figure 2. FEM Working Flow Chart

Here, we choose $w_{1}, w_{2}, w_{3}, w_{4}$ and $w_{5}$ as the test functions which are variants of $f, h, \theta, \Phi$ and $\chi$ respectively. Additionally, the dependent variable $\Theta$ having form $\Theta=$ $\sum_{i=1}^{m} \Theta_{i} S_{i}$ is assumed where $\Theta$ stands for either $f, h, \theta, \Phi$ and $\chi$ with $w_{1}=w_{2}=w_{3}=$ $w_{4}=w_{5}=S_{j},(j=1, \ldots, 5)$. Using both linear and quadratic shape functions, finite element representation is now formulated as:

$$
\left[\begin{array}{lllll}
{\left[M^{11}\right]} & {\left[M^{12}\right]} & {\left[M^{13}\right]} & {\left[M^{14}\right]} & {\left[M^{15}\right]} \\
{\left[M^{21}\right]} & {\left[M^{22}\right]} & {\left[M^{23}\right]} & {\left[M^{24}\right]} & {\left[M^{25}\right]} \\
{\left[M^{31}\right]} & {\left[M^{32}\right]} & {\left[M^{33}\right]} & {\left[M^{34}\right]} & {\left[M^{35}\right]} \\
{\left[M^{41}\right]} & {\left[M^{42}\right]} & {\left[M^{43}\right]} & {\left[M^{44}\right]} & {\left[M^{45}\right]} \\
{\left[M^{51}\right]} & {\left[M^{52}\right]} & {\left[M^{53}\right]} & {\left[M^{54}\right]} & {\left[M^{55}\right]}
\end{array}\right]\left[\begin{array}{c}
f \\
h \\
\theta \\
\Phi \\
\chi
\end{array}\right]=\left[\begin{array}{l}
\left\{t^{1}\right\} \\
\left\{t^{2}\right\} \\
\left\{t^{3}\right\} \\
\left\{t^{4}\right\} \\
\left\{t^{5}\right\}
\end{array}\right]
$$

where $\left[M^{m n}\right],\left\{t^{m}\right\},(m, n)=1, \ldots, 5$ are established as:

$$
\begin{gathered}
M_{i j}^{11}=\int_{\Sigma_{e}} S_{i} S^{\prime}{ }_{j} d \xi, M_{i j}^{12}=-\int_{\Sigma_{e}} S_{i} S_{j} d \xi, M_{i j}^{13}=M_{i j}^{14}=M_{i j}^{15}=0, \\
M_{i j}^{21}=0, M_{i j}^{22}=-\mu_{r} \int_{\Sigma_{e}}(1+2 \gamma \xi) S^{\prime} S^{\prime}{ }_{j} d \xi+\rho_{r} \int_{\Sigma_{e}} \bar{f} S_{i} S^{\prime} d \xi-\rho_{r} \int_{\Sigma_{e}} \bar{h} S_{i} S_{j} d \xi-\sigma_{r} M \int_{\Sigma_{e}} S_{i} S_{j} d \xi, \\
M_{i j}^{23}=0, M_{i j}^{24}=0, M_{i j}^{25}=0, \\
M_{i j}^{31}=M_{i j}^{32}=M_{i j}^{35}=0, M_{i j}^{33}=-k_{r} \int_{\Sigma_{e}}(1+2 \gamma \xi) S_{i}^{\prime} S^{\prime}{ }_{j} d \xi+(\rho c)_{r} \operatorname{Pr} \int_{\Sigma_{e}} \bar{f} S_{i} S^{\prime}{ }_{j} d \xi \\
+\operatorname{Pr} N t \int_{\Sigma_{e}}(1+2 \gamma \xi) S_{i} \bar{\theta}^{\prime} S_{j} d \xi+(\rho c)_{r} \operatorname{Pr} \alpha_{t} \int_{\Sigma_{e}} \overline{f h} S_{i} S_{j} d \xi+(\rho c)_{r} \operatorname{Pr} \alpha_{t} \int_{\Sigma_{e}} \overline{f^{2}} S_{i} S^{\prime}{ }_{j} d \xi, \\
M_{i j}^{34}=\operatorname{Pr} N b \int_{\Sigma_{e}}(1+2 \gamma \xi) S_{i} \bar{\theta}^{\prime} S_{j} d \xi,
\end{gathered}
$$




$$
\begin{aligned}
& M_{i j}^{41}=M_{i j}^{42}=M_{i j}^{45}=0, M_{i j}^{43}=-\frac{N t}{N b} \int_{\Sigma_{e}}(1+2 \gamma \xi) S^{\prime}{ }_{i}{ }^{\prime}{ }_{j} d \xi, \\
& M_{i j}^{44}=-\int_{\Sigma_{e}}(1+2 \gamma \xi) S_{i}^{\prime} S^{\prime}{ }_{j} d \xi+L e \int_{\Sigma_{e}} \bar{f} S_{i} S^{\prime}{ }_{j} d \xi+L e \alpha_{c} \int_{\Sigma_{e}} \overline{f h} S_{i} S_{j} d \xi+L e \alpha_{c} \int_{\Sigma_{e}} \overline{f^{2}} S_{i} S_{j}^{\prime} d \xi \\
& -\operatorname{LeCr} \int_{\Sigma_{e}} S_{i} S_{j} d \xi \\
& M_{i j}^{51}=M_{i j}^{52}=M_{i j}^{53}=0, M_{i j}^{54}=P e \Omega_{1} \int_{\Sigma_{e}}(1+2 \gamma \xi) S^{\prime}{ }_{i} P S^{\prime}{ }_{j} d \xi+P e \int_{\Sigma_{e}}(1+2 \gamma \xi) \bar{\chi} S_{i} P S^{\prime}{ }_{j} d \xi \\
& +P e \int \gamma \bar{\chi} S_{i} P S^{\prime}{ }_{j} d \xi+P e \int \gamma \Omega_{1} S_{i} P S^{\prime}{ }_{j} d \xi, \\
& M_{i j}^{55} \stackrel{\Sigma_{e}}{=}-\int_{\Sigma_{e}}(1+2 \gamma \xi) S^{\prime}{ }_{i} P S^{\prime}{ }_{j} d \xi+L b \int_{\Sigma_{e}} \bar{f} S_{i} P S^{\prime} d \xi-L b C r \int_{\Sigma_{e}} S_{i} S_{j} d \xi . \\
& t_{i}^{1}=0, t_{i}^{2}=-\left((1+2 \xi \gamma) \mu_{r} S_{i} \frac{d h}{d \xi^{\xi}}\right)_{\xi_{e}}^{\xi_{e+1}}-\int_{\Sigma_{e}}\left(M \epsilon+\rho_{r} \epsilon^{2}\right) S_{i} d \xi^{\prime} \\
& t_{i}^{3}=\left(S_{i}\left[-(1+2 \xi \gamma) k_{r}+(\rho c)_{r} \operatorname{Pr} \alpha_{t} f^{2}\right] \frac{d \theta}{d \xi}\right)_{\xi_{e}}^{\xi_{e+1}}, \\
& t_{i}^{4}=\left(S_{i}\left(\left[-(1+2 \xi \gamma) k_{r}+(\rho c)_{r} P r \alpha_{c} f^{2}\right] \frac{d \Phi}{d \zeta}-(1+2 \xi \gamma) \frac{N t}{N b} \frac{d \theta}{d \zeta}\right)\right)_{\xi_{e}}^{\xi_{e+1}}, \\
& t_{i}^{5}=\left(S_{i}\left([-(1+2 \xi \gamma)] \frac{d \chi}{d \xi}+(P e+\Omega)(1+2 \xi \gamma) \frac{d \Phi}{d \xi}\right)\right)_{\xi_{e}}^{\xi_{e+1}} . \\
& \bar{f}=\sum_{i=1}^{m} \overline{f_{i}} S_{i}, \bar{h}=\sum_{i=1}^{m} \overline{h_{i}} S_{i}, \overline{\theta^{\prime}}=\sum_{i=1}^{m} \overline{\theta_{i}} S_{i}^{\prime}, \bar{\chi}=\sum_{i=1}^{m} \overline{\chi_{i}} S_{i}
\end{aligned}
$$

where, $m=2$ corresponds to linear shape function and $m=3$ to quadratic shape function. Similarly, the right hand side column can be evaluated after weak formulation. In this physical configuration, whole domain is alienated into the equal length of both linear and quadratic elements which further solved after implementing the boundary conditions. The

\begin{tabular}{|c|c|c|c|c|c|c|c|c|}
\hline \multirow{2}{*}{ Element Size } & \multicolumn{4}{|c|}{$\xi_{\infty}=6$} & \multicolumn{4}{|c|}{$\xi_{\infty}=8$} \\
\hline & $C f_{r}$ & $N u_{r}$ & $S h_{r}$ & $M_{r}$ & $C f_{r}$ & $N u_{r}$ & $S h_{r}$ & $M_{r}$ \\
\hline 0.1 & 0.995848 & 1.482866 & 2.584126 & 3.731891 & 0.995449 & 1.483016 & 2.584130 & 3.731912 \\
\hline 0.05 & 0.995469 & 1.483159 & 2.576088 & 3.716691 & 0.995070 & 1.483313 & 2.576094 & 3.716716 \\
\hline 0.025 & 0.995375 & 1.483235 & 2.574064 & 3.712841 & 0.994975 & 1.483390 & 2.574071 & 3.712867 \\
\hline 0.01 & 0.995348 & 1.483256 & 2.573496 & 3.711759 & 0.994949 & 1.483412 & 2.573503 & 3.711785 \\
\hline 0.005 & 0.995344 & 1.483259 & 2.573415 & 3.711604 & 0.994945 & 1.483415 & 2.573422 & 3.711630 \\
\hline \multirow{2}{*}{ Element Size } & \multicolumn{4}{|c|}{$\xi_{\infty}=10$} & \multicolumn{4}{|c|}{$\xi_{\infty}=12$} \\
\hline & $C f_{r}$ & $N u_{r}$ & $S h_{r}$ & $M_{r}$ & $C f_{r}$ & $N u_{r}$ & $S h_{r}$ & $M_{r}$ \\
\hline 0.1 & 0.995390 & 1.483037 & 2.584131 & 3.731914 & 0.995380 & 1.483041 & 2.584131 & 3.731915 \\
\hline 0.05 & 0.995011 & 1.483335 & 2.576095 & 3.716720 & 0.995002 & 1.483339 & 2.576095 & 3.716720 \\
\hline 0.025 & 0.994916 & 1.483412 & 2.574072 & 3.712870 & 0.994907 & 1.483416 & 2.574072 & 3.712871 \\
\hline 0.01 & 0.994890 & 1.483434 & 2.573504 & 3.711789 & 0.994880 & 1.483437 & 2.573504 & 3.711789 \\
\hline 0.005 & 0.994886 & 1.483437 & 2.573423 & 3.711634 & 0.994876 & 1.483440 & 2.573423 & 3.711635 \\
\hline
\end{tabular}
Gaussian quadrature method are used to solve the integration maintaining the accuracy of $0.5 \times 10^{-8}$. The desired convergence has been achieved in current problem with optimal element size of 0.01 with boundary layer length of $\xi_{\infty}=10$ which can be observed from Tables 2 and 3. The accuracy of the employed method (Finite Element Method) is also established by direct comparisons. We have compared the results obtained by FEM Results with those of a standard MATLAB built-in function bop $5 c$ (Finite Difference Algorithm) as shown in Table 4 . We have also noticed that MATLAB bop $5 c$ provides convergence only for some set of controlling parameters. Thus, finite element method is advantageous for solving such a complex system.

Table 2. Mesh independency test with linear shape function $(m=2)$ and boundary layer thickness $\left(\xi_{\infty}\right)$. 
Table 3. Mesh independency test with quadratic shape function $(m=3)$ and boundary layer thickness $\left(\xi_{\infty}\right)$.

\begin{tabular}{|c|c|c|c|c|c|c|c|c|}
\hline \multirow{2}{*}{ Element Size } & \multicolumn{4}{|c|}{$\xi_{\infty}=6$} & \multicolumn{4}{|c|}{$\xi_{\infty}=8$} \\
\hline & $C f_{r}$ & $N u_{r}$ & $S h_{r}$ & $M_{r}$ & $C f_{r}$ & $N u_{r}$ & $S h_{r}$ & $M_{r}$ \\
\hline 0.1 & 0.995288 & 1.482829 & 2.573205 & 3.700777 & 0.994889 & 1.482984 & 2.573212 & 3.700801 \\
\hline 0.05 & 0.995329 & 1.483153 & 2.573335 & 3.704312 & 0.994930 & 1.483308 & 2.573342 & 3.704338 \\
\hline 0.025 & 0.995340 & 1.483233 & 2.573374 & 3.707449 & 0.994940 & 1.483389 & 2.573381 & 3.707475 \\
\hline 0.01 & 0.995342 & 1.483256 & 2.573386 & 3.709793 & 0.994943 & 1.483412 & 2.573393 & 3.709819 \\
\hline 0.005 & 0.995343 & 1.483259 & 2.573387 & 3.710653 & 0.994944 & 1.483415 & 2.573394 & 3.710679 \\
\hline \multirow{2}{*}{ Element Size } & \multicolumn{4}{|c|}{$\xi_{\infty}=10$} & \multicolumn{4}{|c|}{$\xi_{\infty}=12$} \\
\hline & $C f_{r}$ & $N u_{r}$ & $S h_{r}$ & $M_{r}$ & $C f_{r}$ & $N u_{r}$ & $S h_{r}$ & $M_{r}$ \\
\hline 0.1 & 0.994830 & 1.483005 & 2.573213 & 3.700804 & 0.994820 & 1.483009 & 2.573213 & 3.700805 \\
\hline 0.05 & 0.994871 & 1.483330 & 2.573343 & 3.704341 & 0.994861 & 1.483334 & 2.573344 & 3.704342 \\
\hline 0.025 & 0.994881 & 1.483411 & 2.573382 & 3.707479 & 0.994872 & 1.483415 & 2.573383 & 3.707480 \\
\hline 0.01 & 0.994884 & 1.483434 & 2.573394 & 3.709823 & 0.994875 & 1.483437 & 2.573394 & 3.709824 \\
\hline 0.005 & 0.994885 & 1.483437 & 2.573395 & 3.710683 & 0.994875 & 1.483440 & 2.573396 & 3.710684 \\
\hline
\end{tabular}

Table 4. Validation of present FEM results with MATLAB bop5c for the parametric values; $\alpha_{t}=\alpha_{c}=M=\epsilon=\beta_{i}(1, \ldots, 4)=\Omega_{1}=\gamma=0.1, N b=N t=0.01, P r=6.2, L e=L b=5$, $C_{r}=P e=1$.

\begin{tabular}{cccccc}
\hline \multirow{2}{*}{$\boldsymbol{s} \boldsymbol{b}$} & \multirow{2}{*}{$\boldsymbol{1}$} & \multicolumn{2}{c}{ FEM } & \multicolumn{2}{c}{ MATLAB $\boldsymbol{b} \boldsymbol{v} \boldsymbol{p 5 c}$} \\
\cline { 3 - 6 } & & $-\boldsymbol{f}^{\prime \prime}(\mathbf{0})$ & $-\boldsymbol{\theta}^{\prime}(\mathbf{0})$ & $-f^{\prime \prime}(\mathbf{0})$ & $-\boldsymbol{\theta}^{\prime}(\mathbf{0})$ \\
\hline-2 & 0.01 & 0.92401060 & 1.71063232 & 0.92401055 & 1.71063225 \\
-1 & & 0.90400142 & 1.54416075 & 0.90400137 & 1.54416073 \\
0 & & 0.88413285 & 1.38255544 & 0.88413281 & 1.38255547 \\
1 & & 0.86454938 & 1.22697477 & 0.86454934 & 1.22697487 \\
2 & & 0.84541153 & 1.07879608 & 0.84541149 & 1.07879626 \\
1 & 0 & 0.88744497 & 1.26305925 & 0.88744493 & 1.26305936 \\
& 0.005 & 0.87633789 & 1.24418922 & 0.87633785 & 1.24418932 \\
& 0.015 & 0.82487969 & 1.21973533 & 0.82487965 & 1.21973542 \\
& 0.02 & 0.81263218 & 1.20750268 & 0.81263215 & 1.20750276 \\
\hline
\end{tabular}

\section{Interpretation of Results}

In the current investigation, nonlinear differential Conditions (13) to (16) with (17) are solved numerically using GFEM. Additionally, the impact of numerous fluid parameters, in particular dimensionless magnetic parameter $M$, velocity slip parameter $\delta_{1}$, curvature parameter $\gamma$, free stream velocity $\epsilon$, initial volume fraction $\phi_{1}$, Brownian motion parameter $\mathrm{Nb}$, Peclet number $\mathrm{Pe}$, thermal slip parameter $\delta_{2}$, chemical reaction parameter $C_{r}$, volume fraction slip parameter $\delta_{3}$, motile concentration parameter $\delta_{4}$ and Stefan blowing $S b$ are addressed and clarified through plots and tables (Tables 5 and 6). For numerical simulation, we have fixed the controlling parameters as $M=0.1$ (for magnetic field less than 0.1 Tesla), $N b=N t=0.01$ ( $<<1$ for $\mathrm{Ag} / \mathrm{MgO}$ hybrid nanoparticles), $\mathrm{Pr}=6.2$ (water as base fluid), $L e=L b=10$ (>>1, high for nanoparticles and algae micro-organisms), $C_{r}=1$ $(>0$, chemical reaction parameter), $\gamma=0.1$ ( $>0$ for cylinder,$=0$ for plate model),$\epsilon=0.1$ (stretching velocity is assumed to be higher than free-stream velocity), $\Omega_{1}=0.1, P e=1$, $\delta_{1}=\delta_{2}=\delta_{3}=\delta_{4}=0.1$ (generally its value $\leq 1$ ) and $\phi_{1}=0.01$ (nanoparticle initial volume fraction not more than $2 \%$ ). The bioconvection parameters can be calculated for alga Chlamydomonas nivalis micro-organism using the data provided in Pedley [47] and Khurana et al. [48]. To sort out the computational non-linearity in the mathematical model, the finite element method (FEM) is executed to settle administering differential conditions, since it gives the flexibility to linearize along with polynomial approximation and shows a very good agreement of convergence in the present study. 
Table 5. Variations in quantities of interest with magnetic field, Stefan blowing, curvature, free stream and initial nanoparticle volume fraction parameters.

\begin{tabular}{|c|c|c|c|c|c|c|c|c|}
\hline$M$ & $S b$ & $\gamma$ & $\epsilon$ & $\phi_{1}$ & $C f_{r}$ & $N u_{r}$ & $S h_{r}$ & $M_{r}$ \\
\hline 0 & 0.1 & 0.1 & 0.1 & 0.01 & 0.958815 & 1.492518 & 2.574126 & 3.724582 \\
\hline 0.2 & & & & & 1.029288 & 1.474745 & 2.572737 & 3.720466 \\
\hline 0.5 & & & & & 1.124164 & 1.450629 & 2.571015 & 3.714981 \\
\hline 1 & & & & & 1.261306 & 1.415459 & 2.568802 & 3.707166 \\
\hline & -3 & & & & 1.048829 & 1.910737 & 2.785927 & 4.166730 \\
\hline & -1 & & & & 1.012783 & 1.622628 & 2.643372 & 3.874408 \\
\hline & 0 & & & & 0.996462 & 1.495597 & 2.579568 & 3.736039 \\
\hline & 1 & & & & 0.981118 & 1.378151 & 2.519442 & 3.602502 \\
\hline & 3 & & & & 0.953033 & 1.168544 & 2.407608 & 3.349496 \\
\hline & & 0 & & & 0.961862 & 1.460638 & 2.545320 & 3.689000 \\
\hline & & 0.2 & & & 1.027009 & 1.506224 & 2.600866 & 3.755347 \\
\hline & & 0.5 & & & 1.118951 & 1.574069 & 2.679831 & 3.850559 \\
\hline & & 1 & & & 1.261265 & 1.683889 & 2.801731 & 3.998840 \\
\hline & & & 0 & & 1.033387 & 1.469748 & 2.572731 & 3.720044 \\
\hline & & & 0.05 & & 1.016872 & 1.475782 & 2.573006 & 3.721086 \\
\hline & & & 0.1 & & 0.994885 & 1.483438 & 2.573403 & 3.722471 \\
\hline & & & 0.2 & & 0.936694 & 1.502411 & 2.574580 & 3.726132 \\
\hline & & & & 0 & 0.906476 & 1.412154 & 2.556206 & 3.715626 \\
\hline & & & & 0.002 & 0.932973 & 1.428518 & 2.559989 & 3.717488 \\
\hline & & & & 0.01 & 0.994885 & 1.483438 & 2.573403 & 3.722471 \\
\hline & & & & 0.02 & 1.144794 & 1.553461 & 2.587661 & 3.730159 \\
\hline
\end{tabular}

Table 6. Variation in quantities of interest with slip parameters, chemical reaction and nanofluid parameters.

\begin{tabular}{|c|c|c|c|c|c|c|c|c|c|c|}
\hline$\delta_{1}$ & $\delta_{2}$ & $\delta_{3}$ & $\delta_{4}$ & $C_{r}$ & $N t$ & $N b$ & $C f_{r}$ & $N u_{r}$ & $S h_{r}$ & $M_{r}$ \\
\hline 0 & 0.1 & 0.1 & 0.1 & 1 & 0.01 & 0.01 & 1.142372 & 1.545323 & 2.584920 & 3.749584 \\
\hline 0.2 & & & & & & & 0.884582 & 1.432896 & 2.564600 & 3.700998 \\
\hline 0.5 & & & & & & & 0.671345 & 1.321554 & 2.546924 & 3.655857 \\
\hline 1 & & & & & & & 0.486269 & 1.203998 & 2.530404 & 3.611454 \\
\hline & 0 & & & & & & 0.994904 & 1.712409 & 2.543340 & 3.713681 \\
\hline & 0.2 & & & & & & 0.994871 & 1.307643 & 2.596794 & 3.729352 \\
\hline & 0.5 & & & & & & 0.994843 & 0.963149 & 2.643405 & 3.743163 \\
\hline & 1 & & & & & & 0.994818 & 0.668176 & 2.684117 & 3.755327 \\
\hline & & 0 & & & & & 0.994276 & 1.465529 & 3.568595 & 4.044178 \\
\hline & & 0.2 & & & & & 0.995230 & 1.493596 & 2.011039 & 3.531668 \\
\hline & & 0.5 & & & & & 0.995718 & 1.508042 & 1.213800 & 3.249761 \\
\hline & & 1 & & & & & 0.996014 & 1.516824 & 0.730580 & 3.072294 \\
\hline & & & 0 & & & & 0.994885 & 1.483438 & 2.573403 & 5.845095 \\
\hline & & & 0.2 & & & & 0.994885 & 1.483438 & 2.573403 & 2.730793 \\
\hline & & & 0.5 & & & & 0.994885 & 1.483438 & 2.573403 & 1.517774 \\
\hline & & & 1 & & & & 0.994885 & 1.483438 & 2.573403 & 0.872117 \\
\hline & & & & 0 & & & 0.995640 & 1.492384 & 1.341482 & 2.486181 \\
\hline & & & & 0.2 & & & 0.995427 & 1.489573 & 1.689495 & 2.852012 \\
\hline & & & & 0.5 & & & 0.995181 & 1.486593 & 2.090375 & 3.255614 \\
\hline & & & & 1 & & & 0.994885 & 1.483438 & 2.573403 & 3.722471 \\
\hline & & & & & 0.001 & & 0.994773 & 1.509164 & 2.757261 & 3.777386 \\
\hline & & & & & 0.005 & & 0.994825 & 1.497691 & 2.672910 & 3.751970 \\
\hline & & & & & 0.01 & & 0.994885 & 1.483438 & 2.573403 & 3.722471 \\
\hline & & & & & 0.05 & & 0.995236 & 1.373002 & 2.000487 & 3.575400 \\
\hline & & & & & & 0.001 & 0.996163 & 1.527152 & 0.488376 & 3.054297 \\
\hline & & & & & & 0.005 & 0.995024 & 1.503198 & 2.346370 & 3.651667 \\
\hline & & & & & & 0.01 & 0.994885 & 1.483438 & 2.573403 & 3.722471 \\
\hline & & & & & & 0.05 & 0.994775 & 1.338567 & 2.753463 & 3.778114 \\
\hline
\end{tabular}




\subsection{Influence of Physical Parameters on Velocity and Temperature Profiles}

Figure 3 shows the velocity profile for magnetic parameter $M$, velocity slip parameter $\delta_{1}$, curvature parameter $\gamma$, free stream velocity $\epsilon$, initial nanoparticle volume fraction $\phi_{1}$ and Stefan blowing $S b$. Figure 3a portrays the influence of magnetic parameter $M$ (0 to 1$)$ over velocity distribution. Lorentz force is delivered in view of presence of an attractive field which opposes the free movement of electrically conducting basefluid (ionized) and it is the main reason behind the diminishing of velocity of nanofluid. Figure $3 \mathrm{~b}$ addresses the velocity profile against slip velocity $\delta_{1}(0$ to 1$)$. Here, it is seen that nanofluid velocity diminishes with the enhancement in slip velocity $\delta_{1}$, may be due to flow velocity near the cylindrical surface is not same as speed of the extending cylinder. Moreover, it is obvious from the figure that all the charts decline particularly up to $\xi=5$ (approximately). Figure $3 \mathrm{c}$ address the profile of velocity for precise sections of curvature parameter $\gamma(0$ to 1$)$. With the increment of $\gamma$, this diagram shows that velocity conveyance increments. Additionally, the cylindrical radius diminishes with expanding estimation of $\gamma$ and subsequently the piece of chamber that is in contact to liquid is scaled down which brings about the decrease of nanoliquid obstruction and in this way the augmentation in profile of velocity is taken note. Figure $3 \mathrm{~d}$ manifests impact of the free stream velocity $\epsilon$ against the velocity distribution for $\epsilon=0-0.4$. For $\epsilon<1$, it signifies the stretching sheet velocity $\left(u_{w}\right)$ is assumed to be more than free-stream velocity $\left(U_{\infty}\right)$. This plot describes that an enormous estimation of $\epsilon$ rises the velocity distribution and is approximately diminished at the surface of cylinder. Impact of initial volume fraction $\phi_{1}(0$ to 0.02$)$ over the velocity is shown by Figure 3e. This graph shows that velocity increases with a very slow speed with the increment in $\phi_{1}$. Additionally, the Figure $3 f$ illustrates the velocity distribution against Stefan blowing parameter $S b$ ( -5 to 5$)$. A critical deviations in profile of velocity can be seen in this figure. With increase in the value of $S b$, velocity increases as depicted in Figure 3f.

Figure 4 illustrates the temperature profile for six crucial parameters. Figure 4 a manifests the temperature distribution against thermal slip flow $\delta_{2}$ ( 0 to 1$)$. This plot illustrates that for higher $\delta_{2}$, reduction in boundary layer thickness has been observed which consequently results in decrease of temperature and the outcome are highly apparent in the boundary layer $0 \leq \xi \leq 1.5$ (approximately).

Figure $4 \mathrm{~b}$ addresses the temperature profile for expanding curvature parameter $\gamma(0-1)$. Here it is seen that temperature ascends with expanding value of $\gamma$ significantly in the boundary region $0.4 \leq \xi \leq 2.5$ (approximately). Additionally, the Figure $4 \mathrm{c}$ illustrates the temperature distribution against Stefan blowing parameter $S b$. A critical deviations in profile of temperature can be seen in this figure. With increase in the value of $S b$, velocity increases as depicted in Figure 4c. The slow impact of initial volume fraction $\phi_{1}$ over the temperature profile can be depicted in Figure $4 \mathrm{~d}$.

Figure 4e shows the impact of Brownian movement boundary $N b$ (0.001 to 0.05 ) over nanoparticle temperature appropriation. At the point when fluid molecules hit with one another, it makes a subjective movement among themself called Brownian movement, which thusly increases the boundary layer thickness and subsequently nanoparticle temperature enhances for additional increment of Brownian movement $\mathrm{Nb}$ which further results in decrement of Nusselt number. Figure $4 \mathrm{f}$ shows the impact of nanoparticle temperature under the influence of thermophoresis $N t$ (0.001 to 0.05$)$. With an expansion in the estimations of thermophoresis parameter $N t$, the temperature inclination tumbles down. 

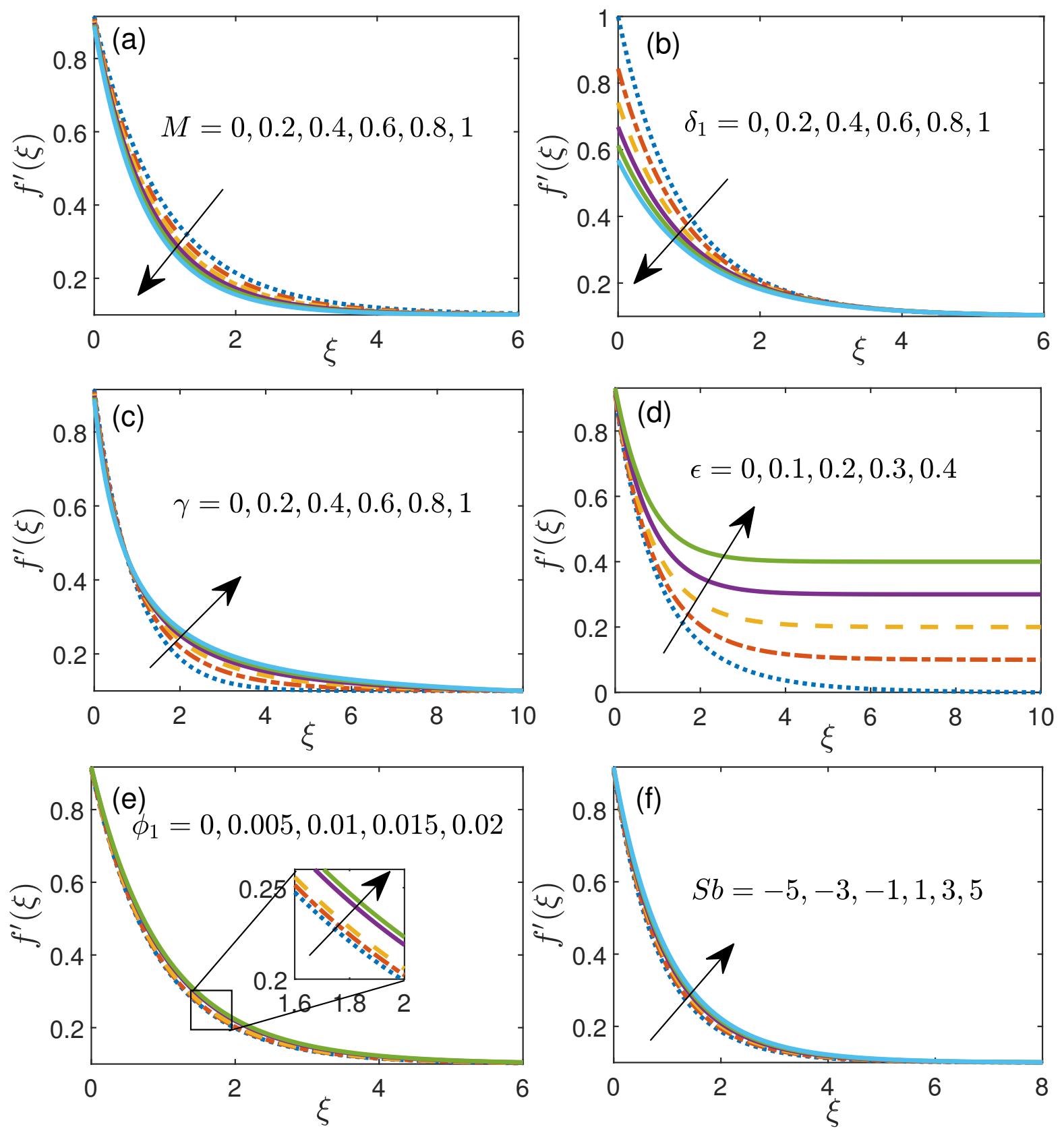

Figure 3. Velocity profile for (a) magnetic parameter $M$, (b) velocity slip parameter $\delta_{1}$, (c) curvature parameter $\gamma$, (d) free stream velocity $\epsilon,(\mathbf{e})$ initial volume fraction $\phi_{1}$ and (f) Stefan blowing $S b$. 

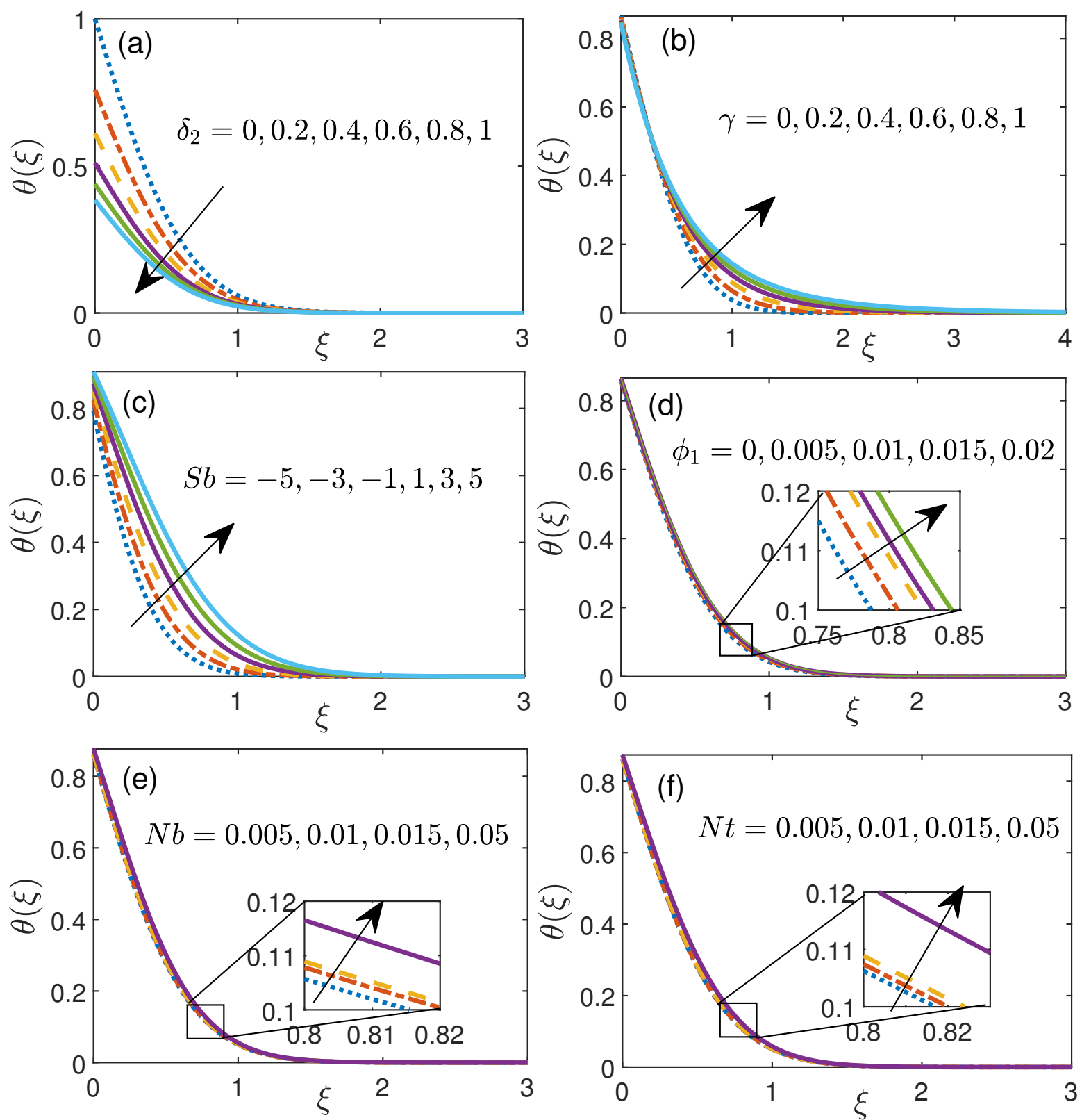

Figure 4. Temperature profile for (a) thermal slip parameter $\delta_{2}$, (b) curvature parameter $\gamma$, (c) Stefan blowing $S b$, (d) initial volume fraction $\phi_{1}$, (e) Brownian motion $\mathrm{Nb}$, (f) thermophoresis $\mathrm{Nt}$.

\subsection{Impact of Controlling Parameters on Nanoparticle Volume Fraction and Motile Density Microorganisms Distribution}

The nanoparticle volume fraction profile for six physical fluid parameters is illustrated via Figure 5. Figure 5a portrays the impact of the slip $\delta_{3}$ ( 0 to 1$)$ on focus conveyance. As we continue expanding the estimation of slip parameter $\delta_{3}$, the liquid fixation diminishes because of the mass slip. The charts are particular inside $0.0 \leq \xi \leq 1.7$ (roughly) and past that area, the end result is not huge. Moreover, the diagrams of fixation are heightened for lower estimations of the $\delta_{3}$. Figure $5 \mathrm{~b}$ represents the nanoparticle volume fraction $\Phi(\xi)$ against curvature parameter $\gamma$ in the range 0 to 1 . With an augmentation in the value of curvature parameter $\gamma$, volume fraction rises as shown in the plot Figure $5 \mathrm{~b}$. As the curvature parameter $(\gamma)$ becomes zero, the present problem is converted to flat stretching sheet problem which justifies the decrement in boundary layer. 

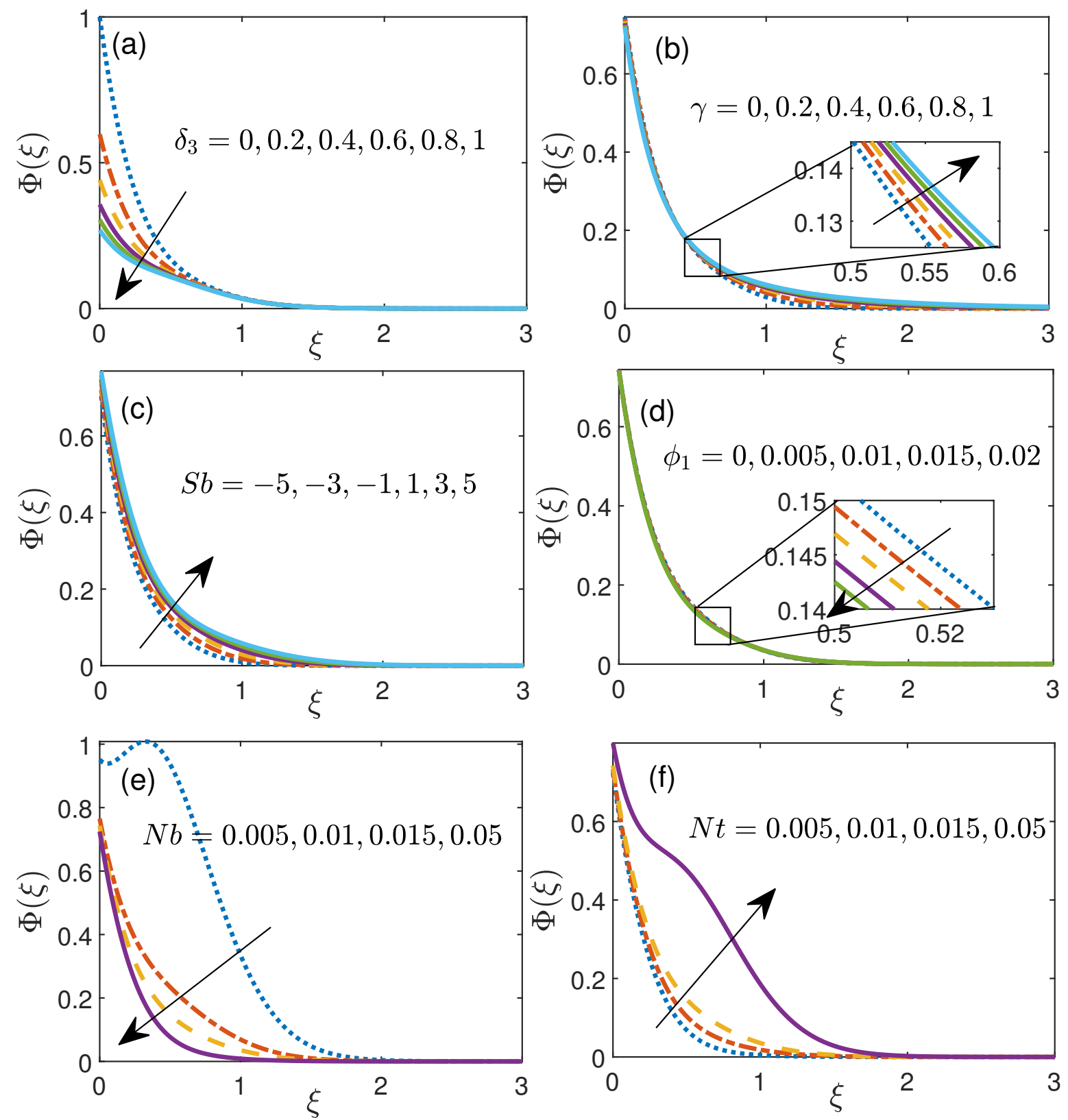

Figure 5. Volume fraction profile for (a) slip parameter $\delta_{3}$, (b) curvature parameter $\gamma$, (c) Stefan blowing $S b$, (d) initial volume fraction $\phi_{1}$, (e) Brownian motion $\mathrm{Nb}$, (f) thermophoresis $\mathrm{Nt}$.

Furthermore, Figure $5 c$ manifests the effect of Stefan blowing parameter $S b$ ( -5 to 5) on nanoparticle volume fraction. Here, this graph shows that, volume fraction enhances with an increment in $S b$. On the other hand, nanoparticle volume fraction declines for higher values of $\phi_{1}$ (0 to 0.02) as depicted in Figure 5d. Figure 5e shows the effect of $\mathrm{Nb}$ (0.001 to 0.05$)$ on profile of nanoparticle volume fraction. With an increment in $\mathrm{Nb}$, nanoparticles slam into one another with higher speed which brings about diminishing of nanoparticle volume fraction and subsequently, the Sherwood number lessens as portrayed in Table 5. Figure $5 \mathrm{f}$ representations variety of nanoparticle volume fraction against $\mathrm{Nt}$. This diagram shows that with an increment in thermophoresis $N t$, nanoparticle volume fraction upgrades. Fundamentally, in the event of thermophoresis applied by a molecule on the other molecule will produce the development of particle movement from more sizzling to colder part and thus strengthening in the nanoparticle volume fraction is noticed through Figure 5f. 
Figure 6a shows the influence of micro-organism slip parameter $\delta_{4}(0$ to 1$)$ over motile concentration. This figure elaborates that motile concentration falls with rise in motile slip parameter $\delta_{4}$. Figure $6 \mathrm{~b}$ manifests the impact of curvature parameter $\gamma(0-1)$ on $\chi(\xi)$. An enhancement in the value of motile concentration is noticed for higher value of curvature parameter $\gamma$. Additionally, motile concentration decreases for higher values of initial volume fraction $\phi_{1}$ and concentration difference parameter $\Omega_{1}$ as shown in Figure $6 \mathrm{c}, \mathrm{d}$ respectively. It is imagined that the non-negative estimations of Peclet number Pe subverts the thickness of gyrotactic micro-organisms since more $\mathrm{Pe}$ improves the movement of liquid particles prompting more slender micro-organisms as seen in Figure 6e. Figure $6 \mathrm{f}$ illustrates motile concentration $\chi(\xi)$ against the chemical reaction parameter $C_{r}(0$ to 1$)$ and this shows that motile concentration falls with rise in chemical reaction parameter $C_{r}$.
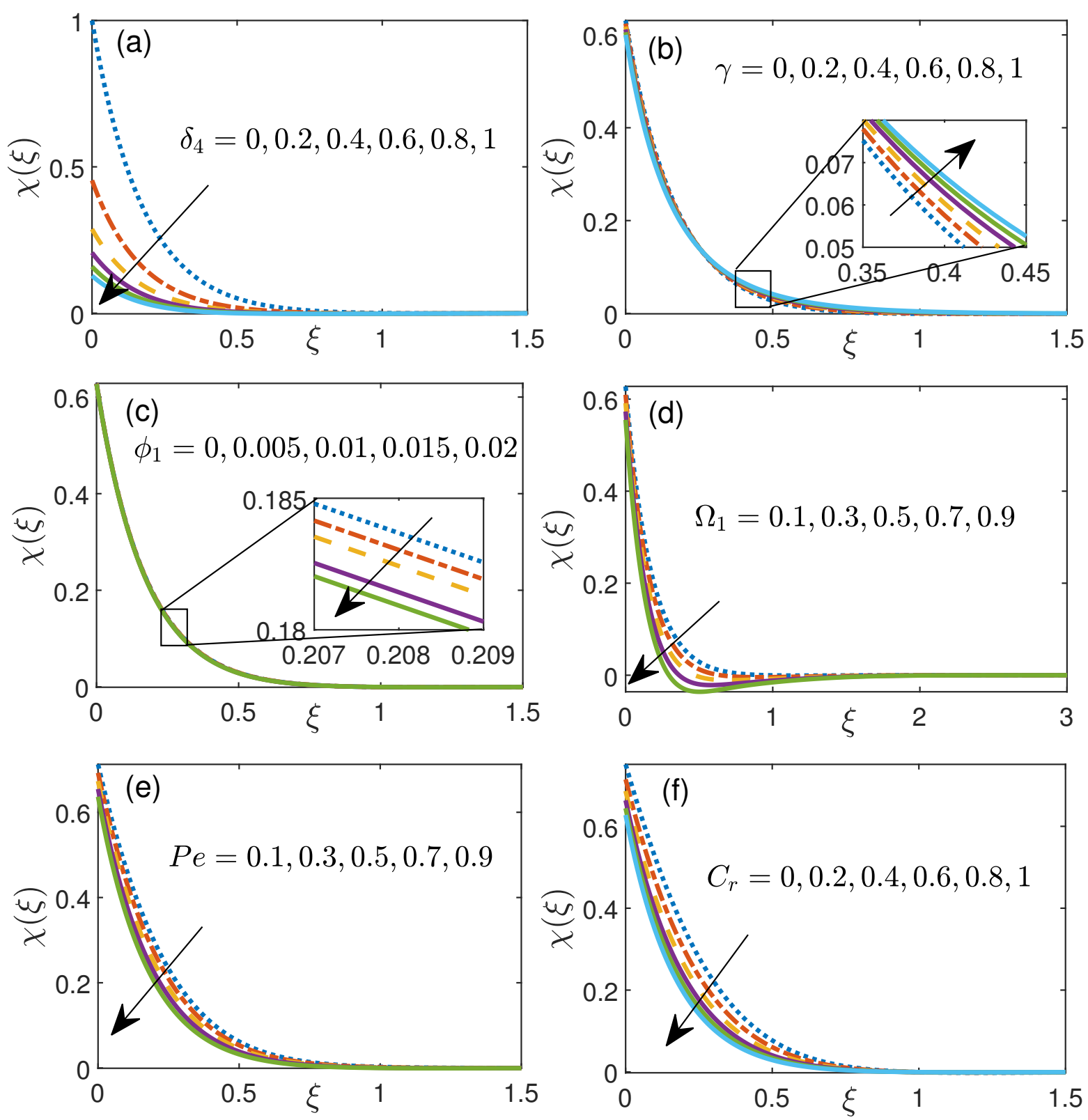

Figure 6. Motile density micro-organism distribution for (a) motile slip parameter $\delta_{4}$, (b) curvature parameter $\gamma$, (c) initial volume fraction $\phi_{1},(\mathbf{d})$ concentration difference parameter $\Omega_{1},(\mathbf{e})$ Peclet number $P e,(\mathbf{f})$ chemical reaction $C_{r}$. 


\subsection{Influence on Skin Friction Coefficient and Nusselt Number with Different Controlling Parameter}

Figure 7a represents the variation of skin friction coefficient $C f_{r}$ against Stefan blowing parameter $S b$ and initial volume fraction $\phi_{1}$ and this figure shows that skin friction coefficient enhances when the values of $S b$ and $\phi_{1}$ rises. Figure $7 \mathrm{~b}$ shows the effect of skin friction coefficient under the influence of velocity slip parameter $\delta_{1}$ and thermal slip parameter $\delta_{2}$. This plot illustrates that skin friction declines with enhancement in $\delta_{1}$ and $\delta_{2}$. Moreover the effect of $\delta_{2}$ is not more prominent in this case as seen in Figure $7 \mathrm{~b}$. While reverse impact is noticed in case of Figure 7c and in this plot, slip parameter plays an important role to increase the value of skin friction coefficient ranging from $0.9944 \leq C f_{r} \leq 0.996$ when volume fraction slip parameter and motile concentration slip parameter are in the range from 0 to 1 . Furthermore, Figure $7 \mathrm{~d}$ manifests that $C f_{r}$ rises with rise in free stream velocity parameter, when $0 \leq \epsilon \leq 0.2$ and curvature parameter, when $0 \leq \gamma \leq 1$.

(a)

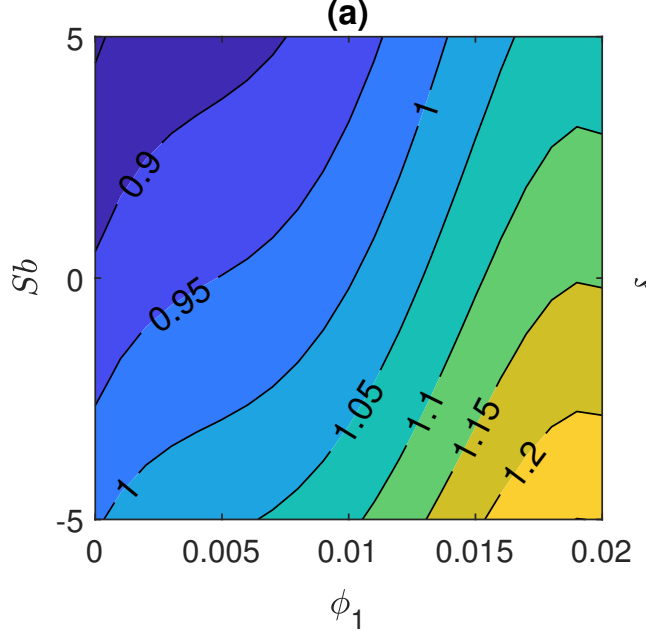

(c)

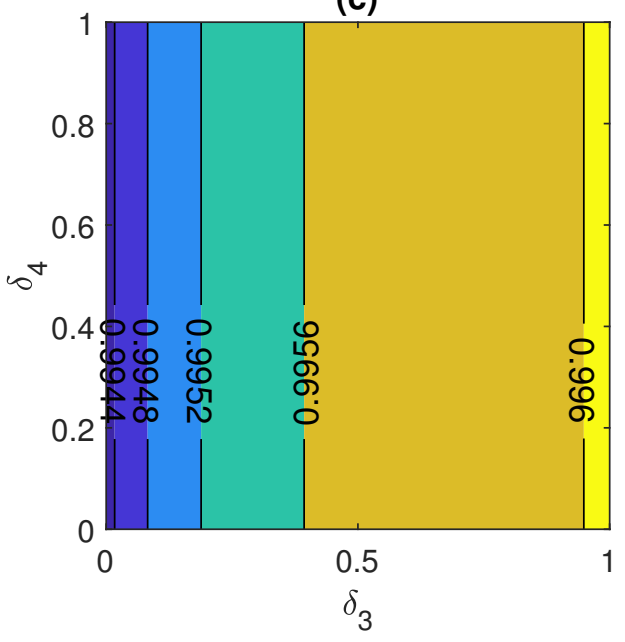

(b)

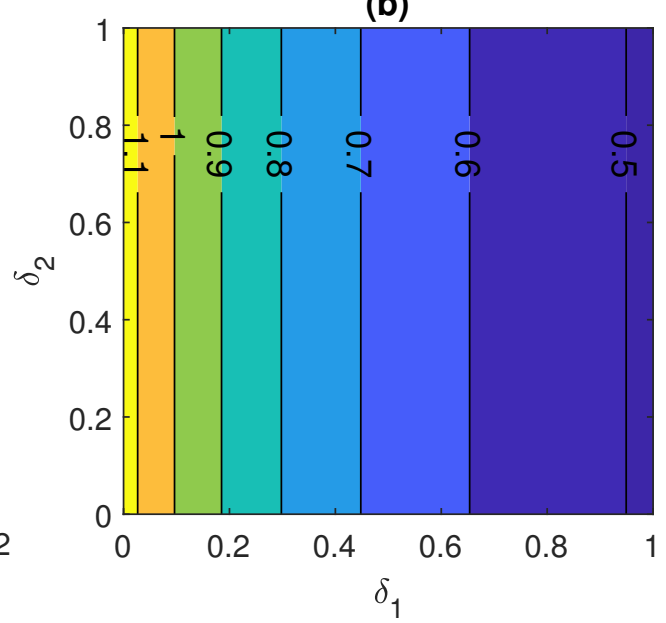

(d)

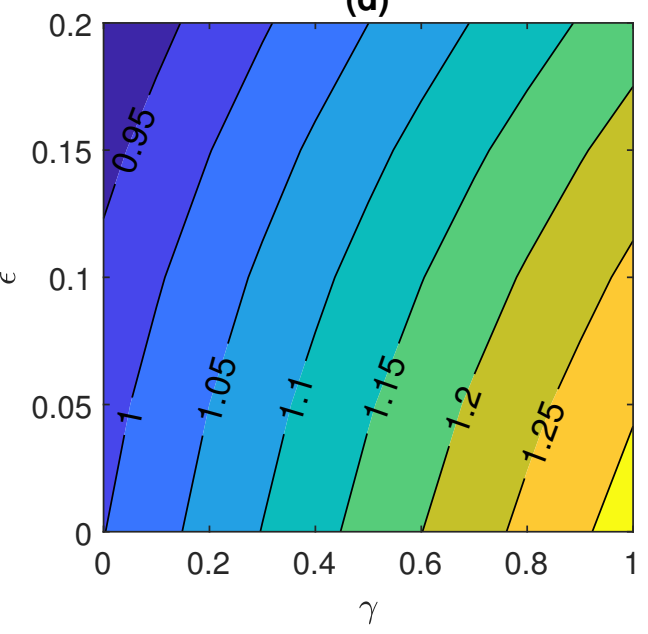

Figure 7. Skin friction coefficient for (a) Stefan blowing $S b$ and initial volume fraction $\phi_{1},(\mathbf{b})$ velocity slip parameter $\delta_{1}$ and thermal slip parameter $\delta_{2}$, (c) volume fraction slip parameter $\delta_{3}$ and microorganism slip parameter $\delta_{4}$ and (d) free stream velocity $\epsilon$ and curvature parameter $\gamma$.

Combined effect of prominent fluid parameters $S b, \phi_{1}$, multiple slips and $N t \& N b$ on heat transfer rate has been illustrated via Figure $8 \mathrm{a}-\mathrm{d}$ respectively. Rate of heat transportation falls with rise in Stefan blowing parameter, when $-5 \leq S b \leq 5$ and initial volume fraction $\phi_{1}$ ranging from 0 to 0.02 . Additionally, this plot shows that the effect of $S b$ is more dominant in comparison to $\phi_{1}$ that can be seen in Figure 8a very clearly. Moreover same pattern is observed in case of Figure $8 \mathrm{~b}$ when combined effect of $0 \leq \delta_{1} \leq 1$ and $0 \leq \delta_{2} \leq 1$ are taken into account. 
Afterthat, Figure $8 \mathrm{c}$ describes the variation in heat transfer under the impact of $\delta_{4}$ and $\delta_{3}$. It is noted from the graph that heat transfer rate increases with only variation in volume fraction slip parameter as a negligible effect is found in case of motile concentration density parameter. Furthermore, the joined impact of higher $N t$ and $N b$ causes the rate of heat transfer to decline as shown in Figure 8d via contour plot.

(a)

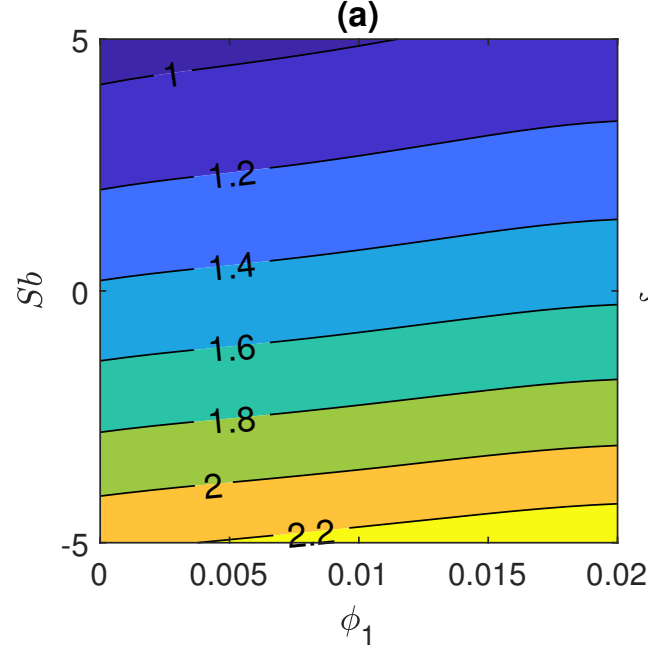

(c)

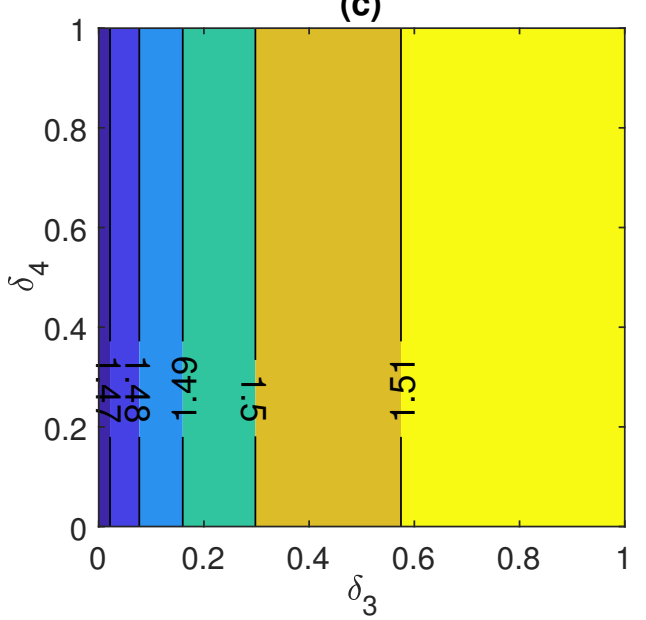

(b)

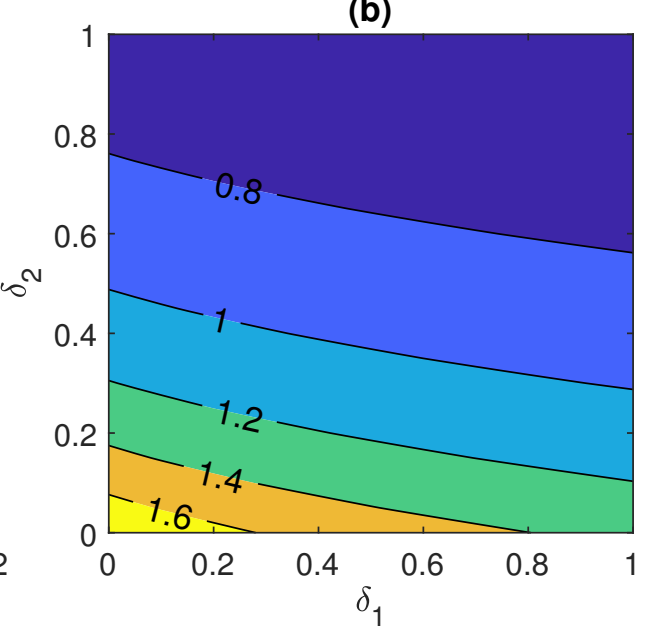

(d)

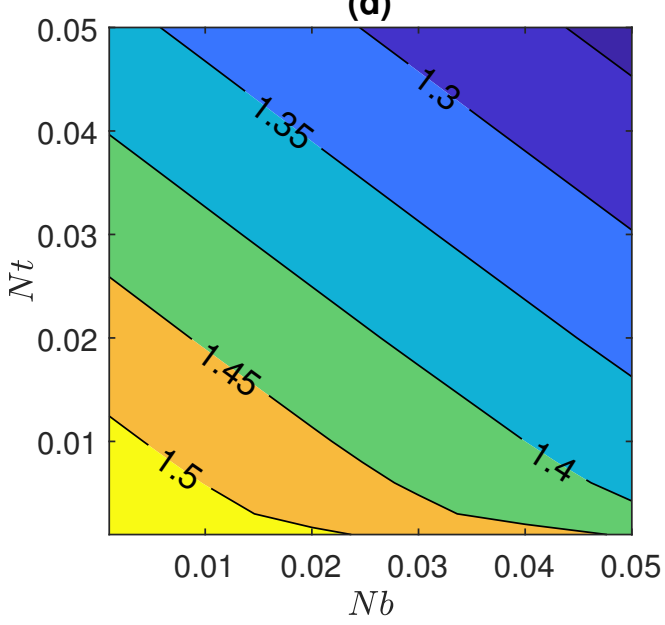

Figure 8. Nusselt number for (a) Stefan blowing $S b$ and initial volume fraction $\phi_{1}$, (b) velocity slip parameter $\delta_{1}$ and thermal slip parameter $\delta_{2}$, (c) volume fraction slip parameter $\delta_{3}$ and motile concentration slip $\delta_{4}$ and (d) Brownian motion $\mathrm{Nb}$ and thermophoresis $\mathrm{Nt}$.

\subsection{Impact of Prominent Physical Parameters on Sherwood Number and Motile Microorganism Number}

Figure 9 depicts the impact of various physical parameters on Sherwood number. The rate of mass transportation declines with the rise in $-5 \leq S b \leq 5$ and $\phi_{1}$ both (as shown in Figure 9a) while it rises with the rise in $\delta_{2}$ and $\delta_{1}$ (as shown in Figure 9b). Additionally, rate of mass transportation declines when combined effect of higher $\delta_{3}$ and $\delta_{4}$ has been taken into account as can be seen in Figure 9c. Further, Figure 9d shows that Sherwood number enhances with enhancement in the values of $C_{r}$ and $\gamma$. Figure 10 represents the motile micro-organism number distribution under the influence of crucial fluid parameters. In this plot, Figure 10a shows that, mass transportation of micro-organisms decreases for higher values of $S b$ and $\phi_{1}$. Furthermore, same impact is noticed in case of higher $\delta_{1}$ and $\delta_{2}$ (see Figure $10 \mathrm{~b}$ ) and in case of higher $\delta_{3}$ and $\delta_{4}$ (see Figure 10c) while reverse impact is noticed for higher $P e$ and $\Omega_{1}$ as shown in Figure 10d. 
(a)

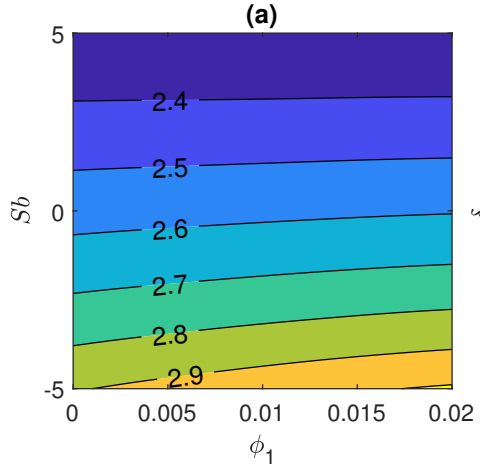

(c)

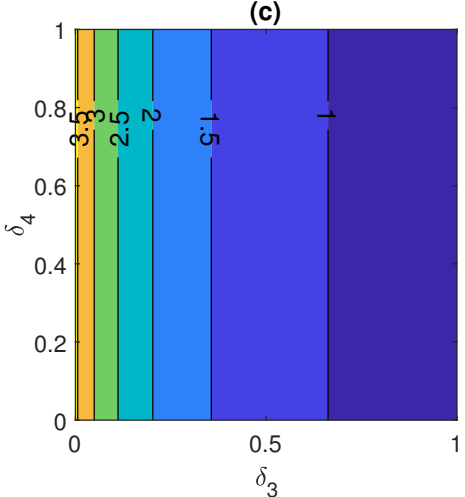

(b)

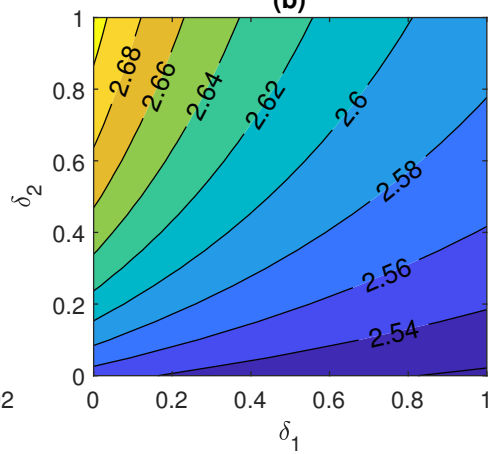

(d)

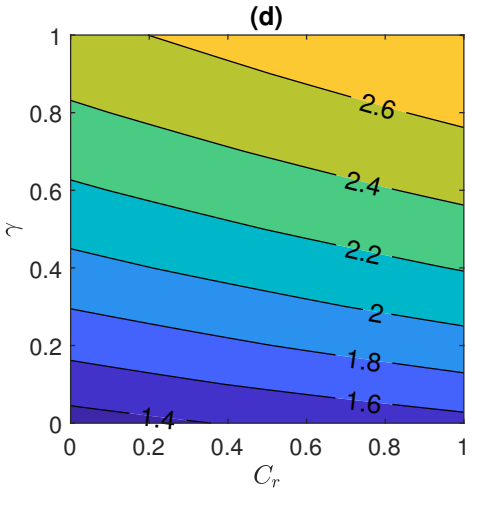

Figure 9. Sherwood number for (a) Stefan blowing $S b$ and initial volume fraction $\phi_{1}$, (b) velocity slip parameter $\delta_{1}$ and thermal slip parameter $\delta_{2}$, (c) volume fraction slip parameter $\delta_{3}$ and motile concentration slip parameter $\delta_{4}$ and (d) Chemical reaction $C_{r}$ and curvature parameter $\gamma$.

(a)

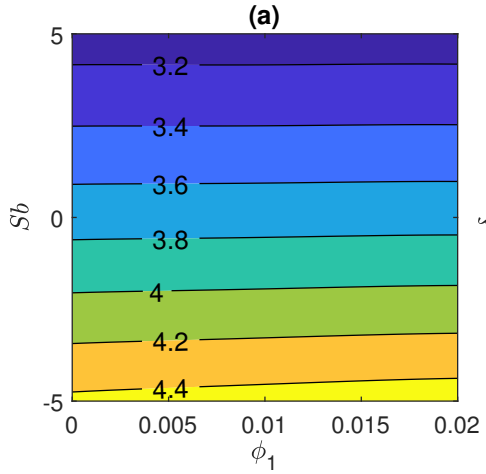

(c)

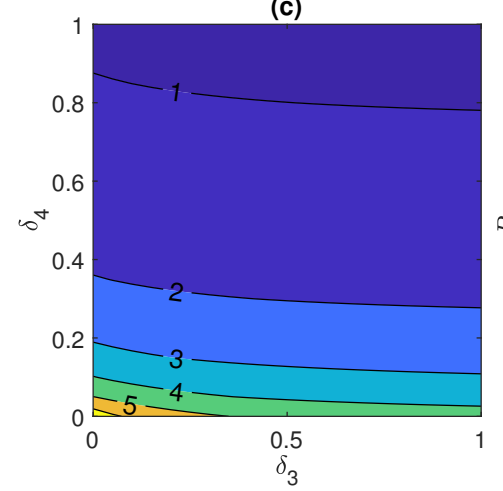

(b)

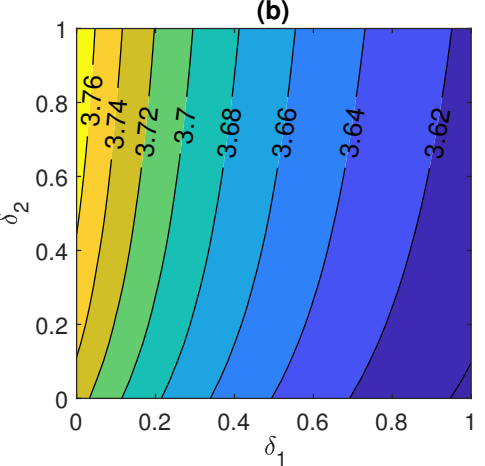

(d)

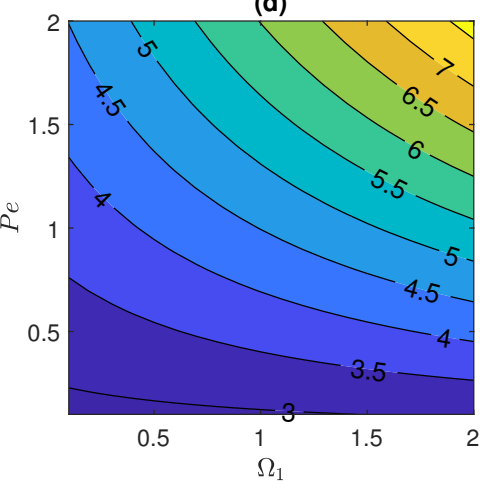

Figure 10. Motile micro-organism density number for (a) Stefan blowing $S b$ and initial volume fraction $\phi_{1},(\mathbf{b})$ velocity slip parameter $\delta_{1}$ and thermal slip parameter $\delta_{2}$, (c) volume fraction slip parameter $\delta_{3}$ and motile concentration slip parameter $\delta_{4}$ and (d) concentration difference parameter $\Omega_{1}$ and Peclet number $P e$. 


\section{Conclusions}

The present study focused on numerical investigation of Stefan blowing MHD hybrid nanofluid flow induced by stretching cylinder by considering Cattaneo-Christov heat flux and mass flux using modified Buongiorno's nanofluid model. The main fallout of the present study incorporating the significance on skin friction and heat transfer is as follows:

1. Stefan blowing and initial nanoparticle volume fraction are found to have maximum impact on skin friction. The optimal (minimum) value of skin friction is recorded for the lower value of initial nanoparticle volume fraction and higher value of Stefan blowing parameter, which is required to have better flow performance and to avoid abrasion.

2. The consideration of velocity slip has a detrimental effect on skin friction to nearly $50 \%$ for the unit increment in its value. However, the skin friction are independent of variation in other slip conditions (thermal, nanoparticle and micro-organism).

3. Higher values of the free stream velocity reduce the skin friction but the curvature parameter has a contrary impact on it.

4. Heat transfer enhancement of upto $20 \%$ is noticed with increment of $2 \%$ of initial volume fraction $\phi_{1}$ of hybrid nanomaterials. With controlled nanoparticle volume fraction, the heat transfer can be optimized required for several industrial processings. Additionally, velocity and thermal slips have considerable impact on the Nusselt number.

5. The nanoparticle volume fraction upsurges with an extended zigzag motion of nanoparticles and the declined thermo-migration of nanoparticles.

6. The curvature parameter and chemically reactive nanoparticles both favor the mass transfer. Even the Sherwood number gets a boost with the increment in initial nanoparticle volume fraction.

7. An excellent agreement is noticed between the numerical results obtained from the Finite Element Method and MATLAB bop5c routine.

The current biological convection model with the involvement of hybrid nanoparticles has many applications in interdisciplinary scientific fields such as biomedicine, biofuel biotechnology, heat exchangers, and enzyme-based biosensors. Our future research will examine rheological properties, especially their impact on microbial transmission, to assess the upcoming announced potential characteristics of new biofuel cells and other technological applications.

Author Contributions: P.R. conceived the nanofluid modelling, P.R. and V.M. conducted the numerical computations and prepared the manuscript, V.M. and G.G. analyzed the results. All authors have read and agreed to the published version of the manuscript.

Funding: This study was supported by Wenzhou Municipal Higher Education Distinctive Program Fund, funded by Wenzhou Education bureau, Wenzhou, China

Conflicts of Interest: The authors declare no conflict of interest.

\section{References}

1. Sakiadis, B.C. Boundary-layer behavior on continuous solid surfaces: I. Boundary-layer equations for two-dimensional and axisymmetric flow. AIChE J. 1961, 7, 26-28. [CrossRef]

2. Choi, S.U.S.; Eastman, J.A. Enhancing thermal conductivity of fluids with nanoparticles. In Proceedings of the ASME International Mechanical Engineering Congress and Exposition, San Diego, CA, USA, 12-17 November 1995.

3. Buongiorno, J. Convective Transport in Nanofluids. J. Heat Transf. 2006, 128, 240-250. [CrossRef]

4. Kuznetsov, A.; Nield, D. Natural convective boundary-layer flow of a nanofluid past a vertical plate: A revised model. Int. J. Therm. Sci. 2014, 77, 126-129. [CrossRef]

5. Dhanai, R.; Rana, P.; Kumar, L. MHD mixed convection nanofluid flow and heat transfer over an inclined cylinder due to velocity and thermal slip effects: Buongiorno's model. Powder Technol. 2016, 288, 140-150. [CrossRef]

6. Swapna, G.; Kumar, L.; Rana, P.; Kumari, A.; Singh, B. Finite element study of radiative double-diffusive mixed convection magneto-micropolar flow in a porous medium with chemical reaction and convective condition. Alex. Eng. J. 2018, 57, 107-120. [CrossRef] 
7. Rana, P.; Bhargava, R.; Bég, O.A.; Kadir, A. Finite element analysis of viscoelastic nanofluid flow with energy dissipation and internal heat source/sink effects. Int. J. Appl. Comput. Math. 2017, 3, 1421-1447. [CrossRef]

8. Vinita, V.; Poply, V. Impact of outer velocity MHD slip flow and heat transfer of nanofluid past a stretching cylinder. Mater. Today Proc. 2020, 26, 3429-3435. [CrossRef]

9. Goyal, R.; Vinita; Sharma, N.; Bhargava, R. GFEM analysis of MHD nanofluid flow toward a power-law stretching sheet in the presence of thermodiffusive effect along with regression investigation. Heat Transf. 2021, 50, 234-256. [CrossRef]

10. Vinita Poply, V.; Goyal, R.; Sharma, N. Analysis of the velocity, thermal, and concentration MHD slip flow over a nonlinear stretching cylinder in the presence of outer velocity. Heat Transf. 2021, 50, 1543-1569. [CrossRef]

11. Vinita Poply, V.; Devi, R. A two-component modeling for free stream velocity in magnetohydrodynamic nanofluid flow with radiation and chemical reaction over a stretching cylinder. Heat Transf. 2020, 50, 3603-3619. [CrossRef]

12. Khan, S.U.; Al-Khaled, K.; Aldabesh, A.; Awais, M.; Tlili, I. Bioconvection flow in accelerated couple stress nanoparticles with activation energy: Bio-fuel applications. Sci. Rep. 2021, 11, 1-15. [CrossRef] [PubMed]

13. Ramzan, M.; Shaheen, N.; Chung, J.D.; Kadry, S.; Chu, Y.M.; Howari, F. Impact of Newtonian heating and Fourier and Fick's laws on a magnetohydrodynamic dusty Casson nanofluid flow with variable heat source/sink over a stretching cylinder. Sci. Rep. 2021, 11, 1-19. [CrossRef] [PubMed]

14. Fourier, J.B.J. Théorie Analytique de la Chaleur; Firmin Didot Père et Fils: Paris, France, 1822.

15. Cattaneo, C. Sulla conduzione del calore. Atti Sem. Mat. Fis. Univ. Modena 1948, 3, 83-101.

16. Christov, C. On frame indifferent formulation of the Maxwell-Cattaneo model of finite-speed heat conduction. Mech. Res. Commun. 2009, 36, 481-486. [CrossRef]

17. Kumar, B.; Seth, G.; Singh, M.; Chamkha, A. Carbon nanotubes (CNTs)-based flow between two spinning discs with porous medium, Cattaneo-Christov (non-Fourier) model and convective thermal condition. J. Therm. Anal. Calorim. 2020. [CrossRef]

18. Abid, N.; Ramzan, M.; Chung, J.D.; Kadry, S.; Chu, Y.M. Comparative analysis of magnetized partially ionized copper, copper oxide-Water and kerosene oil nanofluid flow with Cattaneo-Christov heat flux. Sci. Rep. 2020, 10, 1-14. [CrossRef]

19. Lu, D.; Ramzan, M.; Mohammad, M.; Howari, F.; Chung, J.D. A thin film flow of nanofluid comprising carbon nanotubes influenced by Cattaneo-Christov heat flux and entropy generation. Coatings 2019, 9, 296. [CrossRef]

20. Alamri, S.Z.; Khan, A.A.; Azeez, M.; Ellahi, R. Effects of mass transfer on MHD second grade fluid towards stretching cylinder: A novel perspective of Cattaneo-Christov heat flux model. Phys. Lett. A 2019, 383, 276-281. [CrossRef]

21. Alebraheem, J.; Ramzan, M. Flow of nanofluid with Cattaneo-Christov heat flux model. Appl. Nanosci. 2019, 10, 2989-2999. [CrossRef]

22. Ibrahim, W.; Hindebu, B. Magnetohydrodynamic (MHD) boundary layer flow of eyring-powell nanofluid past stretching cylinder with cattaneo-christov heat flux model. Nonlinear Eng. 2019, 8, 303-317. [CrossRef]

23. Ahmad, S.; Nadeem, S.; Muhammad, N.; Khan, M.N. Cattaneo-Christov heat flux model for stagnation point flow of micropolar nanofluid toward a nonlinear stretching surface with slip effects. J. Therm. Anal. Calorim. 2020, 143, 1187-1199 [CrossRef]

24. Rana, P.; Shukla, N.; Bég, O.A.; Kadir, A.; Singh, B. Unsteady electromagnetic radiative nanofluid stagnation-point flow from a stretching sheet with chemically reactive nanoparticles, Stefan blowing effect and entropy generation. Proc. Inst. Mech. Eng. Part N J. Nanomater. Nanoeng. Nanosyst. 2018, 232, 69-82. [CrossRef]

25. Rana, P.; Shukla, N.; Bég, O.A.; Bhardwaj, A. Lie group analysis of nanofluid slip flow with Stefan blowing effect via modified Buongiorno's Model: Entropy generation analysis. Differ. Equ. Dyn. Syst. 2021, 29, 193-210. [CrossRef]

26. Gowda, R.P.; Kumar, R.N.; Prasannakumara, B.; Nagaraja, B.; Gireesha, B. Exploring magnetic dipole contribution on ferromagnetic nanofluid flow over a stretching sheet: An application of Stefan blowing. J. Mol. Liq. 2021, 335, 116215. [CrossRef]

27. Mabood, F.; Rauf, A.; Prasannakumara, B.; Izadi, M.; Shehzad, S. Impacts of Stefan blowing and mass convention on flow of Maxwell nanofluid of variable thermal conductivity about a rotating disk. Chin. J. Phys. 2021, 71, 260-272. [CrossRef]

28. Gowda, R.P.; Kumar, R.N.; Rauf, A.; Prasannakumara, B.; Shehzad, S. Magnetized flow of sutterby nanofluid through cattaneochristov theory of heat diffusion and stefan blowing condition. Appl. Nanosci. 2021. [CrossRef]

29. Madhukesh, J.; Kumar, R.N.; Gowda, R.P.; Prasannakumara, B.; Ramesh, G.; Khan, M.I.; Khan, S.U.; Chu, Y.M. Numerical simulation of AA7072-AA7075/water-based hybrid nanofluid flow over a curved stretching sheet with Newtonian heating: A non-Fourier heat flux model approach. J. Mol. Liq. 2021, 335, 116103. [CrossRef]

30. Punith Gowda, R.J.; Naveen Kumar, R.; Jyothi, A.M.; Prasannakumara, B.C.; Sarris, I.E. Impact of binary chemical reaction and activation energy on heat and mass transfer of marangoni driven boundary layer flow of a non-Newtonian nanofluid. Processes 2021, 9, 702. [CrossRef]

31. Yusuf, T.A.; Mabood, F.; Prasannakumara, B.; Sarris, I.E. Magneto-bioconvection flow of Williamson nanofluid over an inclined plate with gyrotactic microorganisms and entropy generation. Fluids 2021, 6, 109. [CrossRef]

32. Rana, P.; Dhanai, R.; Kumar, L. MHD slip flow and heat transfer of Al2O3-water nanofluid over a horizontal shrinking cylinder using Buongiorno's model: Effect of nanolayer and nanoparticle diameter. Adv. Powder Technol. 2017, 28, 1727-1738. [CrossRef]

33. Esfe, M.H.; Amiri, M.K.; Alirezaie, A. Thermal conductivity of a hybrid nanofluid. J. Therm. Anal. Calorim. 2018, 134, 1113-1122. [CrossRef]

34. Shah, T.R.; Ali, H.M. Applications of hybrid nanofluids in solar energy, practical limitations and challenges: A critical review. Sol. Energy 2019, 183, 173-203. [CrossRef] 
35. Rana, P. MHD convective heat transfer in the annulus between concentric cylinders utilizing nanoparticles and non-uniform heating. In AIP Conference Proceedings; AIP Publishing LLC: New York, NY, USA, 2020; Volume 2214, p. 020013.

36. Aminian, E.; Moghadasi, H.; Saffari, H. Magnetic field effects on forced convection flow of a hybrid nanofluid in a cylinder filled with porous media: A numerical study. J. Therm. Anal. Calorim. 2020, 141, 2019-2031. [CrossRef]

37. Gul, T.; Khan, A.; Bilal, M.; Alreshidi, N.A.; Mukhtar, S.; Shah, Z.; Kumam, P. Magnetic dipole impact on the hybrid nanofluid flow over an extending surface. Sci. Rep. 2020, 10,1-13.

38. Reddy, M.G.; Rani, M.S.; Kumar, K.G.; Prasannakumar, B.; Lokesh, H. Hybrid dusty fluid flow through a Cattaneo-Christov heat flux model. Phys. A Stat. Mech. Its Appl. 2020, 551, 123975. [CrossRef]

39. Khashi'ie, N.S.; Waini, I.; Zainal, N.A.; Hamzah, K.; Mohd Kasim, A.R. Hybrid nanofluid flow past a shrinking cylinder with prescribed surface heat flux. Symmetry 2020, 12, 1493. [CrossRef]

40. Tassaddiq, A. Impact of Cattaneo-Christov heat flux model on MHD hybrid nano-micropolar fluid flow and heat transfer with viscous and joule dissipation effects. Sci. Rep. 2021, 11, 67. [CrossRef]

41. Shah, Z.; Alzahrani, E.O.; Dawar, A.; Alghamdi, W.; Zaka Ullah, M. Entropy generation in MHD second-grade nanofluid thin film flow containing CNTs with Cattaneo-Christov heat flux model past an unsteady stretching sheet. Appl. Sci. 2020, 10, 2720. [CrossRef]

42. Khan, U.; Ahmad, S.; Hayyat, A.; Khan, I.; Nisar, K.S.; Baleanu, D. On the Cattaneo-Christov heat flux model and OHAM analysis for three different types of nanofluids. Appl. Sci. 2020, 10, 886. [CrossRef]

43. Jakeer, S.; Reddy, P.B.; Rashad, A.; Nabwey, H.A. Impact of heated obstacle position on magneto-hybrid nanofluid flow in a lid-driven porous cavity with Cattaneo-Christov heat flux pattern. Alex. Eng. J. 2021, 60, 821-835. [CrossRef]

44. Esfe, M.H.; Arani, A.A.A.; Rezaie, M.; Yan, W.M.; Karimipour, A. Experimental determination of thermal conductivity and dynamic viscosity of Ag-MgO/water hybrid nanofluid. Int. Commun. Heat Mass Transf. 2015, 66, 189-195. [CrossRef]

45. Ma, Y.; Mohebbi, R.; Rashidi, M.; Yang, Z. MHD convective heat transfer of Ag-MgO/water hybrid nanofluid in a channel with active heaters and coolers. Int. J. Heat Mass Transf. 2019, 137, 714-726. [CrossRef]

46. Selimefendigil, F.; Öztop, H.F. Impact of a rotating cone on forced convection of Ag-MgO/water hybrid nanofluid in a 3D multiple vented T-shaped cavity considering magnetic field effects. J. Therm. Anal. Calorim. 2020, 143, 1485-1501 [CrossRef]

47. Pedley, T.; Hill, N.; Kessler, J. The growth of bioconvection patterns in a uniform suspension of gyrotactic micro-organisms. J. Fluid Mech. 1988, 195, 223-237. [CrossRef] [PubMed]

48. Khurana, M.; Rana, P.; Srivastava, S.; Yadav, S. Magneto-bio-thermal convection in rotating nanoliquid containing gyrotactic microorganism. J. Appl. Comput. Mech. 2021. [CrossRef] 